\title{
A NASH-MOSER THEOREM FOR SINGULAR EVOLUTION EQUATIONS. APPLICATION TO THE SERRE AND GREEN-NAGHDI EQUATIONS
}

\author{
BORYS ALVAREZ-SAMANIEGO AND DAVID LANNES
}

\begin{abstract}
We study the well-posedness of the initial value problem for a wide class of singular evolution equations. We prove a general well-posedness theorem under three assumptions easy to check: the first controls the singular part of the equation, the second the behavior of the nonlinearities, and the third one assumes that an energy estimate can be found for the linearized system. We allow losses of derivatives in this energy estimate and therefore construct a solution by a Nash-Moser iterative scheme. As an application to this general theorem, we prove the well-posedness of the Serre and GreenNaghdi equation and discuss the problem of their validity as asymptotic models for the water-waves equations.
\end{abstract}

\section{INTRODUCTION}

1.1. General setting. We investigate in this paper the local in time well-posedness of singular evolution equations of the form

$$
\left\{\begin{array}{l}
\partial_{t} \underline{u}^{\varepsilon}+\frac{1}{\varepsilon} \mathcal{L}^{\varepsilon}(t) \underline{u}^{\varepsilon}+\mathcal{F}^{\varepsilon}\left[t, \underline{u}^{\varepsilon}\right]=h^{\varepsilon} \\
\underline{u}_{t=0}^{\varepsilon}=\underline{u}_{0}^{\varepsilon},
\end{array}\right.
$$

where $\varepsilon \in\left(0, \varepsilon_{0}\right)$ is a parameter, $\mathcal{L}^{\varepsilon}(t)$ is a linear operator, while $\mathcal{F}^{\varepsilon}[t, \cdot]$ is nonlinear. Under appropriate assumptions, we prove that the initial value problems (IVP) (11) $0<\varepsilon<\varepsilon_{0}$ admit a solution on a time interval $[0, T]$, with $T>0$ independent of $\varepsilon$.

Such a result is known in the case of quasilinear symmetric hyperbolic systems, and provided that the linear (and singular) part $\frac{1}{\varepsilon} \mathcal{L}^{\varepsilon}(t)$ is, say, a constant coefficient anti-adjoint differential operator (see e.g. [19] for the case of classical symmetric system, and [6] for an extension of these results).

In the quasilinear case for instance, an essential step is the study of the IVP associated to the linearization of (11) around any reference function $u$ belonging to some functional space $X$ : if a solution $v$ to this IVP can be found in $X$, and if an energy estimate controls the norm of $v$ in terms of the norm of $u$, then a solution to (1D) can be constructed by a standard Picard iterative scheme.

Our goal here is to investigate situations where this general approach fails. In particular, it sometimes happens that the energy estimate associated to the linearized problem only controls $v$ in a space strictly larger than $X$; when such a loss of information occurs, the standard Picard iterative scheme cannot converge. It is however possible, under certain assumptions, to use the iterative scheme developed by Nash and Moser and used for the first time to solve the embedding problem for

This work was supported by the ACI Jeunes Chercheuses et Jeunes Chercheurs "Dispersion et nonlinéarités". 
Riemannian manifolds [15]. There exists now an extensive literature (e.g. 7, 1]) showing that the technique of Nash and Moser can be used to prove an abstract implicit function theorem.

The implementation of a Nash-Moser iterative scheme is however very technical, and is only used as a last recourse to solve nonlinear evolution equations, though some recent works show that it is a useful tool (e.g. [16, 17, 14, 13, 10, 8]). We develop here a Nash-Moser theorem specific to the general class of IVP (1), which allows us to greatly simplify the general theory (at the cost, sometimes, of optimality - see also [18 for a simplified general Nash-Moser implicit function theorem). The interest of these simplifications is twofold: i) we can state a general well-posedness theorem for (11) under three assumptions easy to check on $\mathcal{L}^{\varepsilon}(t), \mathcal{F}^{\varepsilon}$ and the linearization of (11); ii) we can also handle the presence in the equation of parameters and singular terms. We also show how these results can be used for the justification of asymptotic systems.

As an illustration, we solve the Serre and Green-Naghdi equations which are two of the most widely used models in coastal oceanography (4, 5, 3, and, for instance, [20, 9]). We also address the problem of the relevance of these models as asymptotic models for the exact water-waves equations.

1.2. Organization of the paper. We start by giving the three assumptions of our general well-posedness theorem for (11) in Section 1.4. Section 2 is devoted to the main theorem: it is stated in Section 2.1 and proved in Sections 2.2 and 2.3 In Section [3] we give some generalizations and a corollary of the theorem. The three main assumptions are weakened in Section 3.1 where we allow a more complex dependence of the energy estimate on time derivatives. In Section 3.2, some useful and easy generalizations are given: a slight weakening of the three main assumptions (3.2.2), the possibility of handling other parameters than $\varepsilon$ (3.2.1) and of replacing the linearization of (1) by an approximate linearization (3.2.3). Finally, a corollary is given in Section 3.3, which gives a stability property very useful for the justification of asymptotics to (1).

An application of the main theorem is given in Section 4 where the Serre and Green-Naghdi equations are solved uniformly with respect to the so-called shallowness parameter (Section 4.2). The results of Section 4 are then used in Section 4.3 to address the justification of the Serre and Green-Naghdi models as asymptotic models for the full water-waves equations.

1.3. Notations. - We generically denote by $C\left(\lambda_{1}, \lambda_{2}, \ldots\right)$ a constant depending on the parameters $\lambda_{1}, \lambda_{2}, \ldots$; the dependence on the $\lambda_{j}$ is always assumed to be nondecreasing.

- If $X_{1}$ and $X_{2}$ are two Banach spaces, we denote by $\mathfrak{L}\left(X_{1}, X_{2}\right)$ the set of all continuous linear mappings defined on $X_{1}$ and with values in $X_{2}$.

- If $X$ is a Banach space and $T>0$, then $X_{T}$ stands for $C([0, T] ; X)$, and we denote by $|\cdot|_{X_{T}}$ its canonical norm.

- If $X_{1}$ and $X_{2}$ are two Banach spaces and $\mathcal{F} \in C\left([0, T] ; C^{j}\left(X_{1} ; X_{2}\right)\right)$, we denote by $\mathcal{F}_{u}, \mathcal{F}_{u u}$ and $\mathcal{F}_{(j)}$ the first, second and $j$-th order derivatives of the mapping $u \mapsto \mathcal{F}[\cdot, u]$.

- If $X_{1}$ and $X_{2}$ are two Banach spaces and $\mathcal{F} \in C^{j}\left([0, T] ; C\left(X_{1} ; X_{2}\right)\right)$, we denote by $\mathcal{F}^{(j)}$ the $j$-th order derivative of the mapping $t \mapsto \mathcal{F}[t, \cdot]$.

- We denote $\Lambda:=(1-\Delta)^{1 / 2}$ and $H^{s}\left(\mathbb{R}^{d}\right)(s \in \mathbb{R})$ the usual Sobolev space $H^{s}\left(\mathbb{R}^{d}\right)=$ 
$\left\{u \in \mathcal{S}^{\prime}\left(\mathbb{R}^{d}\right),|u|_{H^{s}}<\infty\right\}$, where $|u|_{H^{s}}=\left|\Lambda^{s} u\right|_{L^{2}}$. We keep this notation if $u$ is a vector or matrix with coefficients in $H^{s}\left(\mathbb{R}^{d}\right)$.

- We use the condensed notation

$$
A_{s}=B_{s}+\left\langle C_{s}\right\rangle_{s>\underline{s}}
$$

to say that $A_{s}=B_{s}$ if $s \leq \underline{s}$ and $A_{s}=B_{s}+C_{s}$ if $s>\underline{s}$.

- By convention, we take $\sum_{j=1}^{0}=0$ and $\prod_{j=1}^{0}=1$.

1.4. Main assumptions. We state here three assumptions which imply the wellposedness of (11). The first one deals with the linear operator $\mathcal{L}^{\varepsilon}$, the second one with the nonlinear term $\mathcal{F}^{\varepsilon}$, and the last one with the well-posedness of the linearization of (11). Throughout this article, we assume that $\left(X^{s}\right)_{s \in \mathbb{R}}$ is a Banach scale in the following sense:

Definition 1. We say that a family of Banach spaces $\left(\left(X^{s}\right),|\cdot|{ }_{s}\right)_{s \in \mathbb{R}}$ is a Banach scale if:

- For all $s \leq s^{\prime}$, one has $X^{s^{\prime}} \subset X^{s}$ and $|\cdot|_{s} \leq|\cdot|_{s^{\prime}}$;

- There exists a family of smoothing operators $\mathcal{S}_{\theta}(\theta \geq 1)$ such that

$$
\forall s<s^{\prime}, \quad \forall u \in X^{s^{\prime}}, \quad\left|\left(1-\mathcal{S}_{\theta}\right) u\right|_{s} \leq C_{s, s^{\prime}} \theta^{s-s^{\prime}}|u|_{s^{\prime}}
$$

and

$\forall s \leq s^{\prime}, \quad \forall u \in X^{s}, \quad \mathcal{S}_{\theta} u \in X^{s^{\prime}} \quad$ and $\quad\left|\mathcal{S}_{\theta} u\right|_{s^{\prime}} \leq C_{s, s^{\prime}} \theta^{s^{\prime}-s}|u|_{s} ;$

- The norms satisfy a convexity property:

$$
\forall s \leq s^{\prime \prime} \leq s^{\prime}, \quad \forall u \in X^{s^{\prime}}, \quad|u|_{s^{\prime \prime}} \leq C_{s, s^{\prime}, s^{\prime \prime}}|u|_{s}^{\mu}|u|_{s^{\prime}}^{1-\mu},
$$

where $\mu$ is given by the relation $\mu s+(1-\mu) s^{\prime}=s^{\prime \prime}$.

The assumption made on the linear operator $\mathcal{L}^{\varepsilon}$ is the following:

Assumption 1. There exist $T>0, s_{0} \in \mathbb{R}$ and $m \geq 0$ such that:

(1) For all $s \geq s_{0}$, one has $\mathcal{L}^{\varepsilon} \in C\left(\mathbb{R} ; \mathfrak{L}\left(X^{s+m} ; X^{s}\right)\right)$ and $\left(\mathcal{L}^{\varepsilon}(\cdot)\right)_{0<\varepsilon<\varepsilon_{0}}$ is bounded in $C\left([0, T] ; \mathfrak{L}\left(X^{s+m} ; X^{s}\right)\right)$;

(2) One can define an evolution operator $U^{\varepsilon}(\cdot) \in C\left(\mathbb{R} ; \mathfrak{L}\left(X^{s}, X^{s}\right)\right) \quad\left(s \geq s_{0}\right)$ as $\forall g \in X^{s}, \quad U^{\varepsilon}(t) g:=u^{\varepsilon}(t), \quad$ where $\quad \partial_{t} u^{\varepsilon}+\frac{1}{\varepsilon} \mathcal{L}^{\varepsilon}(t) u^{\varepsilon}=0, \quad u_{\left.\right|_{t=0} ^{\varepsilon}}^{\varepsilon}=g$, and $\left(U^{\varepsilon}(\cdot)\right)_{0<\varepsilon<\varepsilon_{0}}$ is bounded in $C\left([-T, T] ; \mathfrak{L}\left(X^{s}, X^{s}\right)\right)$.

We can now state our assumption on the nonlinear operator $\mathcal{F}^{\varepsilon}$ :

Assumption 2. There exist $m \geq 0, T>0$, and $s_{0} \in \mathbb{R}$ such that for all $s \geq s_{0}$, $\mathcal{F} \in C\left([0, T] ; C^{2}\left(X^{s+m}, X^{s}\right)\right)$ and:

(1) For all $u \in X^{s+m}$,

$$
\sup _{t \in[0, T]}\left|\mathcal{F}^{\varepsilon}[t, u]\right|_{s} \leq C\left(s, T,|u|_{s_{0}+m}\right)|u|_{s+m}
$$

(2) For all $u, v \in X^{s+m}$ one has

$$
\sup _{t \in[0, T]}\left|\mathcal{F}_{u}^{\varepsilon}[t, u] v\right|_{s} \leq C\left(s, T,|u|_{s_{0}+m}\right)\left(|v|_{s+m}+|u|_{s+m}|v|_{s_{0}+m}\right)
$$


(3) For all $u, v_{1}, v_{2} \in X^{s+m}$ one has

$$
\begin{aligned}
\sup _{t \in[0, T]}\left|\mathcal{F}_{u u}^{\varepsilon}[t, u]\left(v_{1}, v_{2}\right)\right|_{s} \leq & C\left(s, T,|u|_{s_{0}+m}\right)\left(\left|v_{1}\right|_{s+m}\left|v_{2}\right|_{s_{0}+m}+\left|v_{1}\right|_{s_{0}+m}\left|v_{2}\right|_{s+m}\right. \\
& \left.+|u|_{s+m}\left|v_{1}\right|_{s_{0}+m}\left|v_{2}\right|_{s_{0}+m}\right) .
\end{aligned}
$$

Remark 1. The estimates of the assumption are uniform with respect to $\varepsilon \in\left(0, \varepsilon_{0}\right)$ and called tame estimates after Hamilton [7]: the dependence of the r.h.s. on the norms involving the index $s$ is linear.

Before stating the assumption made on the linearization of (1), let us define the space $X_{(j)}^{s}(j \in \mathbb{N})$ and $F^{s}$ as

$$
\begin{aligned}
X_{(j)}^{s} & :=\bigcap_{k=0}^{j} C^{k}\left([0, T] ; X^{s-k m}\right), & |u|_{X_{(j)}^{s}}:=\sum_{k=0}^{j}\left|\left(\varepsilon \partial_{t}\right)^{k} u\right|_{X_{T}^{s-k m}}, \\
F^{s} & :=C\left([0, T] ; X^{s}\right) \times X^{s+m}, & |(f, g)|_{F^{s}}:=|f|_{X_{T}^{s}}+|g|_{s+m}
\end{aligned}
$$

and, for all $(f, g) \in F^{s}$ and $t \in[0, T]$,

$$
\mathcal{I}^{s}(t, f, g):=|g|_{s}+\int_{0}^{t} \sup _{0 \leq t^{\prime \prime} \leq t^{\prime}}\left|f\left(t^{\prime \prime}\right)\right|_{s} d t^{\prime}
$$

Assumption 3. Let $s_{0}, m$ and $T$ be as in Assumption Q There exist $d_{1}, d_{1}^{\prime} \geq 0$ such that for all $s \geq s_{0}+m, u^{\varepsilon} \in X_{(1)}^{s+d_{1}}$ and $\left(f^{\varepsilon}, g^{\varepsilon}\right) \in F^{s+d_{1}^{\prime}}$, the IVP

$$
\partial_{t} v^{\varepsilon}+\frac{1}{\varepsilon} \mathcal{L}^{\varepsilon}(t) v^{\varepsilon}+\mathcal{F}_{u}^{\varepsilon}\left[t, u^{\varepsilon}\right] v^{\varepsilon}=f^{\varepsilon}, \quad v_{\left.\right|_{t=0}}^{\varepsilon}=g^{\varepsilon},
$$

admits a unique solution $v^{\varepsilon} \in C\left([0, T] ; X^{s}\right)$ for all $\varepsilon \in\left(0, \varepsilon_{0}\right)$, and

$$
\begin{aligned}
\left|v^{\varepsilon}\right|_{X_{T}^{s}} & \leq C\left(\varepsilon_{0}, s, T,\left|u^{\varepsilon}\right|_{X_{(1)}^{s_{0}+m+d_{1}}}\right) \\
& \times\left(\mathcal{I}^{s+d_{1}^{\prime}}\left(t, f^{\varepsilon}, g^{\varepsilon}\right)+\left|u^{\varepsilon}\right|_{X_{(1)}^{s+d_{1}}} \mathcal{I}^{s_{0}+m+d_{1}^{\prime}}\left(t, f^{\varepsilon}, g^{\varepsilon}\right)\right) .
\end{aligned}
$$

Remark 2. The above energy estimate exhibits a loss of $d_{1}$ derivatives with respect to the reference state $u^{\varepsilon}$ (and of $d_{1}^{\prime}$ derivatives with respect to the source term and initial data) in the sense that a control of $v^{\varepsilon}$ in $X_{T}^{s}$ requires a control of $u^{\varepsilon}$ in $X_{T}^{s+d_{1}}$. This loss of information makes a standard Picard iterative scheme useless to find a solution to (11). However, since the energy estimate is tame, one can perform a Nash-Moser type iterative scheme. The fact that the energy estimate is also uniform with respect to $\varepsilon \in\left(0, \varepsilon_{0}\right)$ is essential to obtain an existence time $\underline{T}$ independent of $\varepsilon$.

\section{A NASH-Moser TYPe THEOREM}

2.1. Statement of the theorem. We state here the main theorem of this article (a generalization is also given in Theorem 1 below). In the following statement, we use the notations

$\delta:=\max \left\{d_{1}, d_{1}^{\prime}+m\right\}, \quad q:=D-m-d_{1}^{\prime} \quad$ and $\quad P_{\min }:=\delta+\frac{D}{q}(\sqrt{\delta}+\sqrt{2(\delta+q)})^{2}$,

and we also recall that $F^{s+P}=C\left([0, T] ; X^{s+P}\right) \times X^{s+P+m}$. 
Theorem 1. Let $T>0, s_{0}, m, d_{1}$ and $d_{1}^{\prime}$ be such that Assumptions 1 [ 3 are satisfied. Let also $D>\delta, P>P_{\text {min }}, s \geq s_{0}+m$ and $\left(h^{\varepsilon}, \underline{u}_{0}^{\varepsilon}\right)_{0<\varepsilon<\varepsilon_{0}}$ be bounded in $F^{s+P}$. Then there exists $0<\underline{T} \leq T$ and a unique family $\left(\underline{u}^{\varepsilon}\right)_{0<\varepsilon<\varepsilon_{0}}$ bounded in $C\left([0, \underline{T}] ; X^{s+D}\right)$ and solving the IVPs (1) $0<\varepsilon<\varepsilon_{0}$.

2.2. Proof of the theorem. With the evolution operator $U^{\varepsilon}(\cdot)$ defined in Assumption 1, one can define a nonlinear operator $\mathcal{G}^{\varepsilon}[t, \cdot]$ as

$$
\forall t \in[-T, T], \quad \forall u \in X^{s_{0}+m}, \quad \mathcal{G}^{\varepsilon}[t, u]:=U^{\varepsilon}(-t) \mathcal{F}^{\varepsilon}\left[t, U^{\varepsilon}(t) u\right] .
$$

The next lemma shows that one can reduce the study of (1) to the study of

$$
\left\{\begin{array}{l}
\partial_{t} \widetilde{u}^{\varepsilon}+\mathcal{G}^{\varepsilon}\left[t, \widetilde{u}^{\varepsilon}\right]=U^{\varepsilon}(-t) h^{\varepsilon} \\
\widetilde{u}_{\mid t=0}^{\varepsilon}=\underline{u}_{0}^{\varepsilon}
\end{array}\right.
$$

and also states that $\mathcal{G}^{\varepsilon}$ has the same properties as $\mathcal{F}^{\varepsilon}$.

Lemma 1. i. If $\widetilde{u}^{\varepsilon} \in C\left([0, T] ; X^{s}\right)$ solves (7) then $\underline{u}^{\varepsilon} \in C\left([0, T] ; X^{s}\right)$ solves (11), where $\underline{u}^{\varepsilon}(t):=U^{\varepsilon}(t) \widetilde{u}^{\varepsilon}(t)$.

ii. Assumption 国 still holds if one replaces $\mathcal{F}^{\varepsilon}$ by $\mathcal{G}^{\varepsilon}$.

Proof. Assumption 1 shows that if $\widetilde{u}^{\varepsilon} \in C\left([0, T] ; X^{s}\right)$ then $\underline{u}^{\varepsilon} \in C\left([0, T] ; X^{s}\right)$. Remark now that if $\widetilde{u}^{\varepsilon}$ solves (7), then

$$
\partial_{t}\left(U^{\varepsilon}(t) \widetilde{u}^{\varepsilon}\right)=-\frac{1}{\varepsilon} \mathcal{L}^{\varepsilon}(t) U^{\varepsilon}(t) \widetilde{u}^{\varepsilon}-U^{\varepsilon}(t) \mathcal{G}^{\varepsilon}\left[t, \widetilde{u}^{\varepsilon}\right]+h^{\varepsilon}
$$

since $U^{\varepsilon}(t) \mathcal{G}^{\varepsilon}\left[t, \widetilde{u}^{\varepsilon}\right]=\mathcal{F}^{\varepsilon}\left[t, U^{\varepsilon}(t) \widetilde{u}^{\varepsilon}\right]$, the first point of the lemma follows. The second point is a direct consequence of Assumptions 1 and 2 ,

Defining the space $F^{s}$ as in (44) and $E^{s}$ as $C\left([0, T] ; X^{s}\right) \cap C^{1}\left([0, T] ; X^{s-m}\right)$ endowed with its canonical norm (which makes $E^{s}$ different from $X_{(1)}^{s}$ ), we can use Lemma 1, to check that finding a solution $\underline{u}^{\varepsilon}$ to (1) is equivalent to finding a root $\widetilde{u}^{\varepsilon}$ of the equation $\Phi^{\varepsilon}\left(\widetilde{u}^{\varepsilon}\right)=0$, where

$$
\begin{aligned}
& E^{s} \rightarrow \quad F^{s-m} \\
& \Phi^{\varepsilon}: u \mapsto(\underbrace{\partial_{t} u+\mathcal{G}^{\varepsilon}[\cdot, u]-\widetilde{h}^{\varepsilon}}_{:=\Phi_{1}(u)}, u_{\left.\right|_{t=0}}-\underline{u}_{0}^{\varepsilon}),
\end{aligned}
$$

for all $s \geq s_{0}+m$ and $\varepsilon \in\left(0, \varepsilon_{0}\right)$, and with $\widetilde{h}^{\varepsilon}(t):=U^{\varepsilon}(-t) h^{\varepsilon}(t)$.

We seek a root $\widetilde{u}^{\varepsilon}$ to the equation $\Phi^{\varepsilon}\left(\widetilde{u}^{\varepsilon}\right)=0$ as the limit of a Nash-Moser type iterative scheme, namely,

$$
u_{k+1}^{\varepsilon}=u_{k}^{\varepsilon}+S_{k} v_{k}^{\varepsilon},
$$

with $S_{k}:=\mathcal{S}_{\theta_{k}}$, for some $\theta_{k}>0$ to be determined, and where $v_{k}^{\varepsilon}$ solves

$$
\left\{\begin{array}{l}
\partial_{t} v_{k}^{\varepsilon}+\mathcal{G}_{u}^{\varepsilon}\left[t, u_{k}^{\varepsilon}\right] v_{k}^{\varepsilon}=-\Phi_{1}\left(u_{k}^{\varepsilon}\right), \\
\left.v_{k}^{\varepsilon}\right|_{t=0}=\underline{u}_{0}^{\varepsilon}-\left.u_{k}^{\varepsilon}\right|_{t=0} .
\end{array}\right.
$$

The following lemma shows that the above IVP can be solved and that the knowledge of $u_{k}^{\varepsilon}$ thus determines $v_{k}^{\varepsilon}$.

Lemma 2. Suppose that Assumptions[1] [are satisfied, and let $s \geq s_{0}+m$. Assume also that $u_{k}^{\varepsilon} \in E^{s+d_{1}}$ and $\Phi^{\varepsilon}\left(u_{k}^{\varepsilon}\right) \in F^{s+d_{1}^{\prime}}$. Then there exists a unique solution $v_{k}^{\varepsilon} \in E^{s}$ to (9) and

$$
\left|v_{k}^{\varepsilon}\right|_{E^{s}} \leq C\left(\varepsilon_{0}, s, T,\left|u_{k}^{\varepsilon}\right|_{E^{s_{0}+m+d_{1}}}\right)\left(\left|\Phi^{\varepsilon}\left(u_{k}^{\varepsilon}\right)\right|_{F^{s+d_{1}^{\prime}}}+\left|\Phi^{\varepsilon}\left(u_{k}^{\varepsilon}\right)\right|_{F^{s_{0}+m+d_{1}^{\prime}}}\left|u_{k}^{\varepsilon}\right|_{E^{s+d_{1}}}\right) \text {. }
$$


Proof. From Assumption 3, we know that there is a unique solution $w_{k}^{\varepsilon}$ of the IVP

$$
\left\{\begin{array}{l}
\partial_{t} w_{k}^{\varepsilon}+\frac{1}{\varepsilon} \mathcal{L}^{\varepsilon}(t) w_{k}^{\varepsilon}+\mathcal{F}_{u}^{\varepsilon}\left[t, U^{\varepsilon}(t) u_{k}^{\varepsilon}\right] w_{k}^{\varepsilon}=-U^{\varepsilon}(t) \Phi_{1}\left(u_{k}^{\varepsilon}\right) \\
\left.w_{k}^{\varepsilon}\right|_{t=0}=\underline{u_{0}^{\varepsilon}-\left.u_{k}^{\varepsilon}\right|_{t=0}}
\end{array}\right.
$$

as in the proof of Lemma 1, it is easy to check that $v_{k}^{\varepsilon}:=U^{\varepsilon}(-t) w_{k}^{\varepsilon}$ solves (9).

Since Assumption 1 implies that $\left|U^{\varepsilon}(\cdot) u_{k}^{\varepsilon}\right|_{X_{(1)}^{r}} \leq C\left(\varepsilon_{0}\right)\left|u_{k}^{\varepsilon}\right|_{E^{r}}\left(r \geq s_{0}+m\right)$, one can deduce from the estimate of Assumption 3 and Assumption 1 that

$$
\left|v_{k}^{\varepsilon}\right|_{X_{T}^{r}} \leq C\left(\varepsilon_{0}, r, T,\left|u_{k}^{\varepsilon}\right|_{E^{s_{0}+m+d_{1}}}\right)\left(\left|\Phi^{\varepsilon}\left(u_{k}^{\varepsilon}\right)\right|_{F^{r+d_{1}^{\prime}}}+\left|\Phi^{\varepsilon}\left(u_{k}^{\varepsilon}\right)\right|_{F^{s_{0}+m+d_{1}^{\prime}}}\left|u_{k}^{\varepsilon}\right|_{E^{r+d_{1}}}\right) ;
$$

with $r=s$, this is the control we need on $\left|v_{k}^{\varepsilon}\right|_{X_{T}^{s}}$; to conclude the proof, we must therefore show that the same bound holds for $\left|\partial_{t} v_{k}^{\varepsilon}\right|_{X_{T}^{s-m}}$. From the equation one has $\partial_{t} v_{k}^{\varepsilon}=-\mathcal{G}_{u}^{\varepsilon}\left[t, u_{k}^{\varepsilon}\right] v_{k}^{\varepsilon}-\Phi_{1}\left(u_{k}^{\varepsilon}\right)$, so that using Lemma 1,ii, one gets

$$
\left|\partial_{t} v_{k}^{\varepsilon}\right|_{X_{T}^{s-m}} \leq C\left(s, T,\left|u_{k}^{\varepsilon}\right|_{X_{T}^{s_{0}+m}}\right)\left(\left|v_{k}^{\varepsilon}\right|_{X_{T}^{s}}+\left|v_{k}^{\varepsilon}\right|_{X_{T}^{s_{0}+m}}\left|u_{k}^{\varepsilon}\right|_{X_{T}^{s}}\right)+\left|\Phi_{1}\left(u_{k}^{\varepsilon}\right)\right|_{X_{T}^{s-m}} .
$$

and one can conclude with (10) (with $r=s$ and $r=s_{0}+m$ ).

Let us now state the three lemmas which form the heart of the proof, and whose proof is postponed to the next subsections for the sake of clarity.

Lemma 3. Let $D \geq m+d_{1}^{\prime}$ and $s \geq s_{0}+m$. If, for some $M>0$, one has $\left|u_{j}^{\varepsilon}\right|_{E^{s+D}} \leq M(j=k, k+1)$, then

$$
\left|\Phi^{\varepsilon}\left(u_{k+1}^{\varepsilon}\right)\right|_{F^{s+d_{1}^{\prime}}} \leq C(s, T, M)\left(\theta_{k}^{m+d_{1}^{\prime}-D}+\left|v_{k}^{\varepsilon}\right|_{E^{s+D}}\right)\left|v_{k}^{\varepsilon}\right|_{E^{s+D}},
$$

with $C(s, T, M)$ independent of $\varepsilon$.

Lemma 4. Let $D \geq d_{1}$ and $s \geq s_{0}+m$. If, for some $M>0$, one has $\left|u_{k+1}^{\varepsilon}\right|_{E^{s+D}} \leq$ $M$, then

$$
\left|v_{k+1}^{\varepsilon}\right|_{E^{s}} \leq C\left(\varepsilon_{0}, s, T, M\right)\left|\Phi^{\varepsilon}\left(u_{k+1}^{\varepsilon}\right)\right|_{F^{s+d_{1}^{\prime}}}
$$

with $C\left(\varepsilon_{0}, s, T, M\right)$ independent of $\varepsilon$.

Lemma 5. Let $\delta:=\max \left\{d_{1},\left(d_{1}^{\prime}+m\right)\right\}, P \geq D \geq \delta$ and $s \geq s_{0}+m$. If, for some $M>0$, one has $\left|u_{k}^{\varepsilon}\right|_{E^{s+D}} \leq M$ and $\left|\left(h^{\varepsilon}, \underline{u}_{0}^{\varepsilon}\right)\right|_{F^{s+P-m}} \leq M$, then

$$
\left|u_{k+1}^{\varepsilon}\right|_{E^{s+P}} \leq C\left(\varepsilon_{0}, s, T, M\right)\left(1+\theta_{k}^{\delta}\right)\left(1+\left|u_{k}^{\varepsilon}\right|_{E^{s+P}}\right) .
$$

If moreover $\left|u_{k+1}^{\varepsilon}\right|_{E^{s+D}} \leq M$, then one also has

$$
\left|v_{k+1}^{\varepsilon}\right|_{E^{s+P-\delta}} \leq C\left(\varepsilon_{0}, s, T, M\right)\left(1+\left|u_{k+1}^{\varepsilon}\right|_{E^{s+P}}\right) .
$$

We can now proceed with the proof of the theorem, which is a typical Nash-Moser iterative scheme : Lemmas 3 and 4 provide a control of $\left|v_{k+1}^{\varepsilon}\right|_{E^{s}}$ in terms of $\left|v_{k}^{\varepsilon}\right|_{E^{s+D}}$, thus exhibiting a loss of $D$ derivatives but providing a rapid decay of $\left|v_{k+1}^{\varepsilon}\right|_{E^{s}}$, while Lemma 5 control the growth of $\left|v_{k+1}^{\varepsilon}\right|_{E^{s+P-\delta}}$. A control of $\left|v_{k+1}^{\varepsilon}\right|_{E^{s+D}}$ is then recovered by the interpolation formula

$$
\left|v_{k+1}^{\varepsilon}\right|_{E^{s+D}} \leq \operatorname{Cst}\left|v_{k+1}^{\varepsilon}\right|_{E^{s}}^{\mu}\left|v_{k+1}^{\varepsilon}\right|_{E^{s+P-\delta}}^{1-\mu},
$$

with $\mu=1-\frac{D}{P-\delta}$.

Before entering the heart of the proof, let us define the sequence $\left(\theta_{k}\right)_{k}$ used for the smoothing operators as $\theta_{k+1}=\theta_{k}^{r}(k \in \mathbb{N})$, for some $r>1$ defined below.

Remark 3. One has $\theta_{k}=\theta_{0}^{r^{k}}$, so that (if $r>1$ ), $\sum_{k \in \mathbb{N}} \theta_{k}^{-q}=: \underline{\theta}$ converges if and only if $\theta_{0}>1$. Moreover, $\underline{\theta}$ can be made arbitrarily small provided that $\theta_{0}$ is chosen large enough. 
We are now set to control the sequences $\left(u_{k}^{\varepsilon}\right)_{k \in \mathbb{N}}$ and $\left(v_{k}^{\varepsilon}\right)_{k \in \mathbb{N}}$ by induction. For some $M>0$ such that

$$
\left|\left(h^{\varepsilon}, \underline{u}_{0}^{\varepsilon}\right)\right|_{F^{s+P-m}} \leq M \quad \text { and } \quad\left|u_{0}^{\varepsilon}\right|_{E^{s+D}} \leq M / 2,
$$

we define the properties $(\mathrm{i})_{k}-(\mathrm{iii})_{k}$ as

- (i) $)_{k}:\left|u_{k}^{\varepsilon}\right|_{E^{s+P}} \leq \theta_{k}^{\alpha}$;

- (ii) $k:\left|u_{k}^{\varepsilon}\right|_{E^{s+D}} \leq M$;

- (iii) $)_{k}:\left|v_{k}^{\varepsilon}\right|_{E^{s+D}} \leq \theta_{k}^{-q}$, with $q=D-m-d_{1}^{\prime}>0$.

Proof of $(i)_{k+1}-(i i i)_{k+1}$ assuming $(i)_{k}-(i i i)_{k}$. Since one has $\left|u_{k}^{\varepsilon}\right|_{E^{s+D}} \leq M$ by $(\mathrm{ii})_{k}$, $\left|u_{k}^{\varepsilon}\right|_{E^{s+P}} \leq \theta_{k}^{\alpha}$ by $(\mathrm{i})_{k}$ and $\left|\left(h^{\varepsilon}, \underline{u}_{0}^{\varepsilon}\right)\right|_{F^{s+P-m}} \leq M$ by definition of $M$, one can apply Lemma 5 to obtain

$$
\begin{aligned}
\left|u_{k+1}^{\varepsilon}\right|_{E^{s+P}} & \leq C\left(\varepsilon_{0}, s, T, M\right)\left(1+\theta_{k}^{\delta}\right)\left(1+\theta_{k}^{\alpha}\right) \\
& =f\left(\varepsilon_{0}, s, T, M, k\right) \theta_{k+1}^{\alpha}
\end{aligned}
$$

with

$$
f\left(\varepsilon_{0}, s, T, M, k\right)=C\left(\varepsilon_{0}, s, T, M\right)\left(1+\theta_{k}^{\delta}\right)\left(1+\theta_{k}^{\alpha}\right) \theta_{k}^{-\alpha r} .
$$

Assuming that

$$
\delta-\alpha(r-1)<0,
$$

it follows from the explicit expression of $f\left(\varepsilon_{0}, s, T, M, k\right)$ that $f\left(\varepsilon_{0}, s, T, M, k\right) \leq 1$ for all $k \in \mathbb{N}$ provided that $\theta_{0}$ is chosen large enough. This proves (i) $)_{k+1}$.

Recalling that $u_{k+1}^{\varepsilon}=u_{k}^{\varepsilon}+S_{k} v_{k}^{\varepsilon}$, one has $u_{k+1}^{\varepsilon}=u_{0}^{\varepsilon}+\sum_{j=0}^{k} S_{j} v_{j}^{\varepsilon}$, and thus $\left|u_{k+1}^{\varepsilon}\right|_{E^{s+D}} \leq M / 2+\sum_{j=0}^{k} \theta_{k}^{-q}$. As seen in Remark 3, one then gets (ii) $)_{k+1}$ provided that $\theta_{0}$ is chosen large enough.

In order to prove (iii) $)_{k+1}$, remark first that it follows from Lemmas 3 and 4 and the choice of the sequence $\left(\theta_{k}\right)_{k \in \mathbb{N}}$ that

$$
\left|v_{k+1}^{\varepsilon}\right|_{E^{s}} \leq C\left(\varepsilon_{0}, s, T, M\right) \theta_{k+1}^{-2 q / r} .
$$

We can also use the second assertion of Lemma 5 to obtain

$$
\left|v_{k+1}^{\varepsilon}\right|_{E^{s+P-\delta}} \leq C\left(\varepsilon_{0}, s, T, M\right)\left(1+\theta_{k+1}^{\alpha}\right) .
$$

It follows therefore from (11), (14) and (15) that

$$
\begin{aligned}
\left|v_{k+1}^{\varepsilon}\right|_{E^{s+D}} & \leq C\left(\varepsilon_{0}, s, T, M\right) \theta_{k+1}^{-2 \mu q / r}\left(1+\theta_{k+1}^{\alpha}\right)^{1-\mu} \\
& =g\left(\varepsilon_{0}, s, T, M, k\right) \theta_{k+1}^{-q},
\end{aligned}
$$

with $g\left(\varepsilon_{0}, s, T, M, k\right):=C\left(\varepsilon_{0}, s, T, M\right) \theta_{k+1}^{-2 \mu q / r}\left(1+\theta_{k+1}^{\alpha}\right)^{1-\mu} \theta_{k+1}^{q}$. Choosing $r$ such that

$$
1<r<\frac{2 \mu q}{q+\alpha(1-\mu)},
$$

one gets that $g\left(\varepsilon_{0}, s, T, M, k\right) \leq 1$ for all $k \in \mathbb{N}$, provided that $\theta_{0}$ is chosen large enough.

It follows from the lines above that in order to complete the proof of the heredity of the induction property, we just have to take $\theta_{0}$ large enough, and to prove that one can choose $\alpha, r$ and $P$ such that the conditions (13) and (16) are satisfied. This is done in the following lemma:

Lemma 6. Let $\alpha=\delta+\sqrt{2 \delta(\delta+q)}$; if $P>\delta+\frac{D}{q}(\sqrt{\delta}+\sqrt{2(\delta+q)})^{2}$, there exists $r>1$ such that conditions (13) and (16) are satisfied. 
Proof. Let us denote $\underline{r}:=\frac{2 \mu q}{q+\alpha(1-\mu)}$. Quite obviously, (13) and (16) are satisfied with $r=\underline{r}-\epsilon(\epsilon>0$ small enough), provided that $\underline{r}-1>\delta / \alpha$, that is,

$$
\frac{(2 q+\alpha) \mu-(q+\alpha)}{q+\alpha(1-\mu)}>\frac{\delta}{\alpha},
$$

or equivalently, if

$$
\mu>1-\frac{q(1-\delta / \alpha)}{2 q+\alpha+\delta}=: \mu_{\min }(\alpha) .
$$

The value of $\alpha$ given in the statement of the lemma corresponds to the minimum of $\mu_{\min }(\alpha)$. One then computes that $\mu_{\min }(\alpha)=1-\frac{q}{(\sqrt{\delta}+\sqrt{2(\delta+q)})^{2}}$, and the lemma then follows from the observation that $\mu>\mu_{\min }(\alpha)$ is equivalent to $P>\delta+$ $\frac{D}{1-\mu_{\min }}$.

Proof of $(i)_{0}-(i i i)_{0}$. We have to construct here the first term of the sequence $u_{0}^{\varepsilon}$ in such a way that $(\mathrm{i})_{0}-(\mathrm{iii})_{0}$ and (12) are satisfied for some $M>0$ and $\theta_{0}>0$. We need the following lemma:

Lemma 7. For all $s \geq s_{0}+m$ and $\left(h^{\varepsilon}, \underline{u}_{0}^{\varepsilon}\right) \in F^{s+P}$, there exists $u_{0}^{\varepsilon} \in E^{s+P}$ such that $\left.u_{0}^{\varepsilon}\right|_{t=0}=\underline{u}_{0}^{\varepsilon}$ and such that

$$
\left|u_{0}^{\varepsilon}\right|_{E^{s+D}} \leq C\left(s, T,\left|\left(h^{\varepsilon}, \underline{u}_{0}^{\varepsilon}\right)\right|_{F^{s+D}}\right) \quad \text { and } \quad\left|u_{0}^{\varepsilon}\right|_{E^{s+P}} \leq C\left(s, T,\left|\left(h^{\varepsilon}, \underline{u}_{0}^{\varepsilon}\right)\right|_{F^{s+P}}\right) \text {, }
$$

and

$$
\left|\Phi^{\varepsilon}\left(u_{0}^{\varepsilon}\right)\right|_{F^{s+D+d_{1}^{\prime}}} \leq T C\left(s, T,\left|\left(h^{\varepsilon}, \underline{u}_{0}^{\varepsilon}\right)\right|_{F^{s+D+d_{1}^{\prime}+m}}\right) .
$$

Proof. Let us define $u_{0}^{\varepsilon} \in C\left([0, T] ; X^{s+P}\right)$ as

$$
u_{0}^{\varepsilon}(t)=\underline{u}_{0}^{\varepsilon}+\int_{0}^{t}\left(\widetilde{h}^{\varepsilon}\left(t^{\prime}\right)-\mathcal{G}^{\varepsilon}\left[t^{\prime}, \underline{u}_{0}^{\varepsilon}\right]\right) d t^{\prime}
$$

From Lemma 1 and the definition of $|\cdot|_{E^{s}}$, one gets for all $r \geq 0$,

$$
\left|u_{0}^{\varepsilon}\right|_{E^{s+r}} \leq\left|\underline{u}_{0}^{\varepsilon}\right|_{s+r}+C\left(s, T,\left|\underline{u}_{0}^{\varepsilon}\right|_{s_{0}+m}\right)\left(\left|h^{\varepsilon}\right|_{X_{T}^{s+r}}+\left|\underline{u}_{0}^{\varepsilon}\right|_{s+r+m}\right) ;
$$

the estimates on $u_{0}^{\varepsilon}$ given in the lemma are thus a consequence of (17), with $r=D$ and $r=P$.

By definition of $\Phi^{\varepsilon}$, one also has

$$
\begin{aligned}
\Phi^{\varepsilon}\left(u_{0}^{\varepsilon}\right) & =\left(\mathcal{G}^{\varepsilon}\left[\cdot, u_{0}^{\varepsilon}\right]-\mathcal{G}^{\varepsilon}\left[\cdot, \underline{u}_{0}^{\varepsilon}\right], 0\right) \\
& =\left(\int_{0}^{1} \mathcal{G}_{u}^{\varepsilon}\left[\cdot, \underline{u}_{0}^{\varepsilon}+z\left(u_{0}^{\varepsilon}-\underline{u}_{0}^{\varepsilon}\right)\right]\left(u_{0}^{\varepsilon}-\underline{u}_{0}^{\varepsilon}\right) d z, 0\right),
\end{aligned}
$$

so that one deduces from Assumptions 1 and 2 that

$$
\left|\Phi^{\varepsilon}\left(u_{0}^{\varepsilon}\right)\right|_{F^{s+D+d_{1}^{\prime}}} \leq C\left(s, T,\left|u_{0}^{\varepsilon}\right|_{X_{T}^{s+D+d_{1}^{\prime}+m}},\left|\underline{u}_{0}^{\varepsilon}\right|_{s+D+d_{1}^{\prime}+m}\right)\left|u_{0}^{\varepsilon}-\underline{u}_{0}^{\varepsilon}\right|_{X_{T}^{s+D+d_{1}^{\prime}+m}},
$$

and the estimate on $\Phi^{\varepsilon}\left(u_{0}^{\varepsilon}\right)$ of the lemma follows easily.

Thanks to the lemma, taking $M=M\left(s, T,\left|\left(h^{\varepsilon}, \underline{u}_{0}^{\varepsilon}\right)\right|_{F^{s+D}}\right)$ large enough, one gets $\left|u_{0}^{\varepsilon}\right|_{E^{s+D}} \leq M / 2$, which proves (ii) $)_{0}$. Choosing $\theta_{0}=\theta_{0}\left(s, T,\left|\left(h^{\varepsilon}, \underline{u}_{0}^{\varepsilon}\right)\right|_{F^{s+P}}\right)$ large enough, one also gets (i) from Lemma 7 . In order to prove (iii) $)_{0}$, remark first that Lemma 2 yields $\left|v_{0}^{\varepsilon}\right|_{E^{s+D}} \leq C\left(\varepsilon_{0}, s, T, M\right)\left|\Phi^{\varepsilon}\left(u_{0}^{\varepsilon}\right)\right|_{F^{s+D}+d_{1}^{\prime}}\left(1+\theta_{0}^{\alpha}\right)$. It follows therefore from the lemma that, taking a smaller $T$ if necessary, (iii) $)_{0}$ is satisfied, which ends the induction proof of properties $(\mathrm{i})_{k},(\mathrm{ii})_{k}$ and (iii) $)_{k}$. 
The end of the existence part of the proof of the theorem is now straightforward: it follows from $(\mathrm{i})_{k},(\mathrm{ii})_{k}$ and (iii) $)_{k}$ that the series $u_{0}^{\varepsilon}+\sum_{k} S_{k} v_{k}^{\varepsilon}$ converges to some $u^{\varepsilon} \in E^{s+D}$ and taking the limit $k \rightarrow \infty$ in Lemma 3 shows that $\Phi^{\varepsilon}\left(u^{\varepsilon}\right)=0$.

In order to conclude the proof of the theorem, we must now prove that the solution constructed above is unique. Assuming that $u^{\varepsilon, j} \in E^{s+D}(j=1,2)$ are both solutions to (1), we show that $w:=u^{\varepsilon, 2}-u^{\varepsilon, 1}$ is identically 0 . Let us remark that $w$ solves the IVP

$$
\left\{\begin{array}{l}
\partial_{t} w+\frac{1}{\varepsilon} \mathcal{L}^{\varepsilon}(t) w+\mathcal{F}_{u}^{\varepsilon}\left[t, u^{\varepsilon, 2}\right] w=H \\
w_{\mid t=0}=0
\end{array}\right.
$$

with $H:=\mathcal{F}^{\varepsilon}\left[t, u^{\varepsilon, 1}\right]-\mathcal{F}^{\varepsilon}\left[t, u^{\varepsilon, 2}\right]-\mathcal{F}_{u}^{\varepsilon}\left[t, u^{\varepsilon, 2}\right]\left(u^{\varepsilon, 1}-u^{\varepsilon, 2}\right)$.

A direct application of Assumption 3 yields

$$
|w(t)|_{s_{0}+m} \leq C\left(\varepsilon_{0}, T,\left|u^{\varepsilon, 1}\right|_{\left.X_{(1)}^{s_{0}+m+\delta}\right)} \int_{0}^{t} \sup _{0 \leq t^{\prime \prime} \leq t^{\prime}}\left|H\left(t^{\prime \prime}\right)\right|_{s_{0}+m+d_{1}^{\prime}} d t^{\prime},\right.
$$

and since $\left|H\left(t^{\prime}\right)\right|_{s_{0}+m+d_{1}^{\prime}} \leq C\left(s, T,\left|u^{\varepsilon, 2}\right|_{X_{T}^{s_{0}+m+\delta}}\right)\left|w\left(t^{\prime}\right)\right|_{s_{0}+m}$ by Assumption 2, 3], a Gronwall argument shows that $w=0$.

\subsection{Proof of Lemmas 3, 4, 5.}

2.3.1. Proof of Lemma 3. In order to give an upper bound for $\left|\Phi^{\varepsilon}\left(u_{k+1}^{\varepsilon}\right)\right|_{F^{s+d_{1}^{\prime}}}$, we need to control $\Phi_{1}\left(u_{k+1}^{\varepsilon}\right)$ in $X_{T}^{s+d_{1}^{\prime}}$ and $\left|u_{k+1||_{t=0}^{\varepsilon}}^{\varepsilon}-\underline{u}_{0}^{\varepsilon}\right|_{s+d_{1}^{\prime}+m}$.

First remark that a second order Taylor expansion of $\Phi_{1}\left(u_{k+1}^{\varepsilon}\right)$ yields

$$
\begin{aligned}
\Phi_{1}\left(u_{k+1}^{\varepsilon}\right)= & \Phi_{1}\left(u_{k}^{\varepsilon}\right)+\Phi_{1}^{\prime}\left(u_{k}^{\varepsilon}\right)\left(u_{k+1}^{\varepsilon}-u_{k}^{\varepsilon}\right) \\
& +\int_{0}^{1}(1-z) \Phi_{1}^{\prime \prime}\left(u_{k}^{\varepsilon}+z\left(u_{k+1}^{\varepsilon}-u_{k}^{\varepsilon}\right)\right)\left(u_{k+1}^{\varepsilon}-u_{k}^{\varepsilon}, u_{k+1}^{\varepsilon}-u_{k}^{\varepsilon}\right) d z .
\end{aligned}
$$

Since by (요 $)$, one has $u_{k+1}^{\varepsilon}-u_{k}^{\varepsilon}=S_{k} v_{k}^{\varepsilon}$, and since by definition $\Phi_{1}\left(u_{k}^{\varepsilon}\right)=\partial_{t} u_{k}^{\varepsilon}+$ $\mathcal{G}^{\varepsilon}\left[\cdot, u_{k}^{\varepsilon}\right]-\widetilde{h}^{\varepsilon}$, it follows that

$$
\Phi_{1}\left(u_{k+1}^{\varepsilon}\right)=E_{1}+E_{2},
$$

with

$$
\begin{aligned}
E_{1}= & \Phi_{1}\left(u_{k}^{\varepsilon}\right)+\partial_{t} v_{k}^{\varepsilon}+\mathcal{G}_{u}^{\varepsilon}\left[\cdot, u_{k}^{\varepsilon}\right] v_{k}^{\varepsilon} \\
& +\int_{0}^{1}(1-z) \mathcal{G}_{u u}^{\varepsilon}\left[\cdot, u_{k}^{\varepsilon}+z\left(u_{k+1}^{\varepsilon}-u_{k}^{\varepsilon}\right)\right]\left(S_{k} v_{k}^{\varepsilon}, S_{k} v_{k}^{\varepsilon}\right) d z \\
= & \int_{0}^{1}(1-z) \mathcal{G}_{u u}^{\varepsilon}\left[\cdot, u_{k}^{\varepsilon}+z\left(u_{k+1}^{\varepsilon}-u_{k}^{\varepsilon}\right)\right]\left(S_{k} v_{k}^{\varepsilon}, S_{k} v_{k}^{\varepsilon}\right) d z
\end{aligned}
$$

(the last equality stemming from the fact that $v_{k}^{\varepsilon}$ solves (9)), and

$$
E_{2}=\left(S_{k}-1\right) \partial_{t} v_{k}^{\varepsilon}+\mathcal{G}_{u}^{\varepsilon}\left[\cdot, u_{k}^{\varepsilon}\right]\left(\left(S_{k}-1\right) v_{k}^{\varepsilon}\right) .
$$

Since by Lemma 1]ii, $\mathcal{G}^{\varepsilon}$ satisfies Assumption [2], and since $s+d_{1}^{\prime}+m \leq s+D$, one can control $E_{1}$ as

$$
\begin{aligned}
\left|E_{1}\right|_{X_{T}^{s+d_{1}^{\prime}}} & \leq C\left(s, T,\left|u_{k}^{\varepsilon}\right|_{X_{T}^{s+D}},\left|u_{k+1}^{\varepsilon}\right|_{X_{T}^{s+D}}\right)\left|S_{k} v_{k}^{\varepsilon}\right|_{X_{T}^{s+D}}^{2} \\
& \leq C(s, T, M)\left|v_{k}^{\varepsilon}\right|_{X_{T}^{s+D}}^{2}
\end{aligned}
$$


Since Lemma 1,ii also ensures that $\mathcal{G}^{\varepsilon}$ satisfies Assumption 22 2, one gets

$$
\left|\mathcal{G}_{u}^{\varepsilon}\left[\cdot, u_{k}^{\varepsilon}\right]\left(\left(S_{k}-1\right) v_{k}^{\varepsilon}\right)\right|_{X_{T}^{s+d_{1}^{\prime}}} \leq C(s, T, M) \sup _{t \in[0, T]}\left|\left(S_{k}-1\right) v_{k}^{\varepsilon}(t)\right|_{s+m+d_{1}^{\prime}} .
$$

It is then a consequence of the properties of the regularizing operators (recall that $\left.S_{k}=\mathcal{S}_{\theta_{k}}\right)$, that

$$
\begin{aligned}
\left|E_{2}\right|_{X_{T}^{s+d_{1}^{\prime}}} & \leq \operatorname{Cst} \theta_{k}^{m+d_{1}^{\prime}-D}\left(\left|\partial_{t} v_{k}^{\varepsilon}\right|_{X_{T}^{s+D-m}}+C(s, T, M)\left|v_{k}^{\varepsilon}\right|_{X_{T}^{s+D}}\right) \\
& \leq C(s, T, M) \theta_{k}^{m+d_{1}^{\prime}-D}\left|v_{k}^{\varepsilon}\right|_{E^{s+D}} .
\end{aligned}
$$

It is then a simple consequence of (18), (21) and (22) to conclude that

$$
\left|\Phi_{1}\left(u_{k+1}^{\varepsilon}\right)\right|_{X_{T}^{s+d_{1}^{\prime}}} \leq C(s, T, M)\left(\theta_{k}^{m+d_{1}^{\prime}-D}+\left|v_{k}^{\varepsilon}\right|_{E^{s+D}}\right)\left|v_{k}^{\varepsilon}\right|_{E^{s+D}} .
$$

We now turn to control $\left|u_{k+1}^{\varepsilon}\right|_{t=0}-\left.\underline{u}_{0}^{\varepsilon}\right|_{s+d_{1}^{\prime}+m}$. Since $\left.u_{k+1}^{\varepsilon}\right|_{t=0}-\underline{u}_{0}^{\varepsilon}=\left.\left(S_{k}-1\right) v_{k}^{\varepsilon}\right|_{t=0}$, one gets

$$
\begin{aligned}
\left|u_{k+1||_{t=0}^{\varepsilon}}^{\varepsilon}-\underline{u}_{0}^{\varepsilon}\right|_{s+d_{1}^{\prime}+m} & \leq \operatorname{Cst} \theta_{k}^{m+d_{1}^{\prime}-D} \sup _{t \in[0, T]}\left|v_{k}^{\varepsilon}(t)\right|_{s+D} \\
& \leq \operatorname{Cst} \theta_{k}^{m+d_{1}^{\prime}-D}\left|v_{k}^{\varepsilon}\right|_{E^{s+D}}
\end{aligned}
$$

The lemma follows directly from (23) and (24).

2.3.2. Proof of Lemma 4. Since $\left|u_{k+1}^{\varepsilon}\right|_{E^{s+d_{1}}} \leq M$, one gets therefore from Lemma 2 (at step $k+1)$,

$$
\left|v_{k+1}^{\varepsilon}\right|_{E^{s}} \leq C\left(\varepsilon_{0}, s, T, M\right)\left|\Phi^{\varepsilon}\left(u_{k+1}^{\varepsilon}\right)\right|_{F^{s+d_{1}^{\prime}}},
$$

and the lemma is proved.

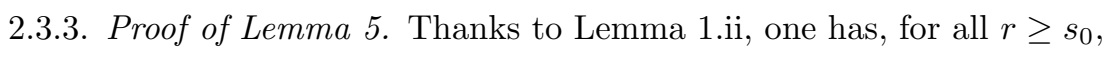

$$
\left|\Phi^{\varepsilon}(u)\right|_{F^{r}} \leq C\left(|u|_{E^{s_{0}+m}}\right)|u|_{E^{r+m}}+\text { Cst }\left|\left(h^{\varepsilon}, \underline{u}_{0}^{\varepsilon}\right)\right|_{F^{r}} ;
$$

remark also that since $u_{k+1}^{\varepsilon}=u_{k}^{\varepsilon}+S_{k} v_{k}^{\varepsilon}$, one can use the properties of the regularizing operator $S_{k}=\mathcal{S}_{\theta_{k}}$ to obtain

$$
\left|u_{k+1}^{\varepsilon}\right|_{E^{s+P}} \leq\left|u_{k}^{\varepsilon}\right|_{E^{s+P}}+\operatorname{Cst} \theta_{k}^{\delta}\left|v_{k}^{\varepsilon}\right|_{E^{s+P-\delta}} .
$$

From Lemma 2, one deduces

$$
\left|v_{k}^{\varepsilon}\right|_{E^{s+P-\delta}} \leq C\left(\varepsilon_{0}, s, T, M\right)\left(\left|\Phi^{\varepsilon}\left(u_{k}^{\varepsilon}\right)\right|_{F^{s_{0}+m+d_{1}^{\prime}}}\left|u_{k}^{\varepsilon}\right|_{E^{s+P}}+\left|\Phi^{\varepsilon}\left(u_{k}^{\varepsilon}\right)\right|_{F^{s+P-m}}\right)
$$

so that, using (26) with $r=s_{0}+m+d_{1}^{\prime}$ and $r=s+P-m$, and the assumption made on $\left(h^{\varepsilon}, \underline{u}_{0}^{\varepsilon}\right)$, one obtains

$$
\left|v_{k}^{\varepsilon}\right|_{E^{s+P-\delta}} \leq C\left(\varepsilon_{0}, s, T, M\right)\left(1+\left|u_{k}^{\varepsilon}\right|_{E^{s+P}}\right) .
$$

Together with (27), this last estimate shows that

$$
\left|u_{k+1}^{\varepsilon}\right|_{E^{s+P}} \leq C\left(\varepsilon_{0}, s, T, M\right)\left(1+\theta_{k}^{\delta}\right)\left(1+\left|u_{k}^{\varepsilon}\right|_{E^{s+P}}\right),
$$

so that the proof of the first assertion is complete.

The last part of the lemma is exactly (28) with the index $k$ replaced by $k+1$. 


\section{Further Results}

We propose in this section a more general version of Theorem 1 and some remarks extending its range of validity. We also a stability property very useful for the justification of asymptotic models for instance.

3.1. A more general version of Theorem 1, The aim of this section is to prove a result similar to Theorem 1 when the energy estimates of Assumption 3 involve $p+1(p \geq 1)$ time derivatives of the reference solution $u^{\varepsilon}$ (such a situation occurs for instance with the water-waves equations). With this goal in mind, we replace the three assumptions 103 by generalizations to the case $p \neq 0$. We first generalize the spaces $E^{s}$ and $F^{s}$ used in the proof of Theorem 11 as follows:

$$
E_{(p+1)}^{s}:=\bigcap_{i=0}^{p+1} C^{i}\left([0, T] ; X^{s-i m}\right), \quad F_{(p)}^{s}:=\bigcap_{i=0}^{p} C^{i}\left([0, T] ; X^{s-i m}\right) \times X^{s+m}
$$

endowed with the norms

$$
\begin{array}{r}
|u|_{E_{(p+1)}^{s}}=|u|_{X_{T}^{s}}+\left|\partial_{t} u\right|_{X_{T}^{s-m}}+\sum_{i=1}^{p}\left|\left(\varepsilon \partial_{t}\right)^{i} \partial_{t} u\right|_{X_{T}^{s-(i+1) m}}, \\
|(f, g)|_{F_{(p)}^{s}}=|f|_{X_{T}^{s}}+|g|_{s+m}+\sum_{i=1}^{p}\left|\left(\varepsilon \partial_{t}\right)^{i} f\right|_{X_{T}^{s-i m}}
\end{array}
$$

and we also define for all $(f, g) \in F_{(p)}^{s}$ and $t \in[0, T]$,

$$
\mathcal{I}_{(p)}^{s}(t, f, g)=|g|_{s+m}+\int_{0}^{t} \sum_{i=0}^{p} \sup _{0 \leq t^{\prime \prime} \leq t^{\prime}}\left|\left(\varepsilon \partial_{t}\right)^{i} f\left(t^{\prime \prime}\right)\right|_{s-i m} d t^{\prime}
$$

(so that $E_{(1)}^{s}, F_{(0)}^{s}$ and $\mathcal{I}_{(0)}^{s}$ coincide with $E^{s}, F^{s}$ and $\mathcal{I}^{s}$ respectively).

Assumption 1'. Assumption 11 holds with (1) replaced by the stronger condition (when $p \geq 1$ ):

(11)' For all $s \geq s_{0}$, one has $\mathcal{L}^{\varepsilon} \in C^{p}\left(\mathbb{R} ; \mathfrak{L}\left(X^{s+m} ; X^{s}\right)\right)$ and for all $i=0, \ldots, p$, $\left(\varepsilon^{i} \frac{d^{i}}{(d t)^{2}} \mathcal{L}^{\varepsilon}(\cdot)\right)_{0<\varepsilon<\varepsilon_{0}}$ is bounded in $C\left([0, T] ; \mathfrak{L}\left(X^{s+m} ; X^{s}\right)\right)$.

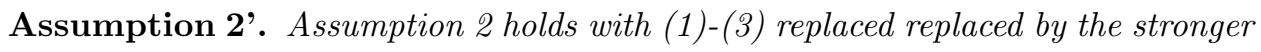
conditions: For all $0 \leq i \leq p$ and $0 \leq i+j \leq p+2$, and for all $s \geq s_{0}+i m$, one has $\mathcal{F}^{\varepsilon} \in C^{i}\left([0, T] ; C^{j}\left(X^{s+m}, X^{s-i m}\right)\right)$ and

$$
\begin{aligned}
& \sup _{t \in[0, T]}\left|\varepsilon^{i} \mathcal{F}_{(j)}^{\varepsilon(i)}[t, u]\left(v_{1}, \ldots, v_{j}\right)\right|_{s-i m} \leq C\left(s, T,|u|_{s_{0}+(i+1) m}\right) \\
& \quad \times\left(\sum_{k=1}^{j}\left|v_{k}\right|_{s+m} \prod_{l \neq k}\left|v_{l}\right|_{s_{0}+(i+1) m}+|u|_{s+m} \prod_{k=1}^{j}\left|v_{k}\right|_{s_{0}+(i+1) m}\right) .
\end{aligned}
$$

Assumption 3'. There exists $p \in \mathbb{N}$ such that for all $s \geq s_{0}+m, u^{\varepsilon} \in X_{(p+1)}^{s+d_{1}}$ and $\left(f^{\varepsilon}, g^{\varepsilon}\right) \in F_{(p)}^{s+d_{1}^{\prime}}$, the IVPs (6) $)_{0<\varepsilon<\varepsilon_{0}}$ admit a unique solution $v^{\varepsilon} \in C\left([0, T] ; X^{s}\right)$, and

$$
\begin{aligned}
\forall t \in[0, T], \quad\left|v^{\varepsilon}\right|_{X_{T}^{s}} & \leq C\left(\varepsilon_{0}, s, T,\left|u^{\varepsilon}\right|_{\left.X_{(p+1)}^{s_{0}+m+d_{1}}\right)}\right. \\
& \times\left(\mathcal{I}_{(p)}^{s+d_{1}^{\prime}}\left(t, f^{\varepsilon}, g^{\varepsilon}\right)+\left|u^{\varepsilon}\right|_{X_{(p+1)}^{s+d_{1}}} \mathcal{I}_{(p)}^{s_{0}+m+d_{1}^{\prime}}\left(t, f^{\varepsilon}, g^{\varepsilon}\right)\right) .
\end{aligned}
$$


Theorem 1 then admits the following generalization (with $\delta$ and $P_{\min }$ as defined in Theorem 10:

Theorem 1'. Let $p \in \mathbb{N}, T>0, s_{0}, m, d_{1}$ and $d_{1}^{\prime}$ be such that Assumptions [1' 3 ' are satisfied. Let also $D>\delta, P>P_{\text {min }}, s \geq s_{0}+(p+1) m$ and $\left(h^{\varepsilon}, \underline{u}_{0}^{\varepsilon}\right)_{0<\varepsilon<\varepsilon_{0}}$ be bounded in $F_{(p)}^{s+P}$.

Then there exists $0<\underline{T} \leq T$ and a unique family $\left(\underline{u}^{\varepsilon}\right)_{0<\varepsilon<\varepsilon_{0}}$ bounded in $C\left([0, \underline{T}] ; X^{s+D}\right)$ and solving the IVPs (1) $0<\varepsilon<\varepsilon_{0}$.

Proof. The proof is a generalization of the proof of Theorem 1, and we just sketch the adaptations to be done.

The second property of Lemma 1 can be generalized as follows:

Lemma 1'. Assumption [2' still holds if one replaces $\mathcal{F}^{\varepsilon}$ by $\mathcal{G}^{\varepsilon}$.

Proof. Let us first prove the following fact (recalling that the normed space $X_{(i)}^{s}$ is defined in (3) ): for $0 \leq i \leq p$ and $s \geq s_{0}+i m$,

$$
\forall f \in X_{(i)}^{s}, \quad U^{\varepsilon}(\cdot) f \in X_{(i)}^{s} \quad \text { and } \quad\left|U^{\varepsilon}(\cdot) f\right|_{X_{(i)}^{s}} \leq C(s, T)|f|_{X_{(i)}^{s}}
$$

indeed, from the definition of the evolution operator $U^{\varepsilon}(\cdot)$, one can check that $\left(\varepsilon \partial_{t}\right)^{i}\left(U^{\varepsilon}(\cdot) f(\cdot)\right)$ is a sum of terms of the form

$$
\left(\mathcal{L}^{\varepsilon}\right)^{\alpha_{0}}\left(\varepsilon \partial_{t} \mathcal{L}^{\varepsilon}\right)^{\alpha_{1}} \ldots\left(\varepsilon^{i-1} \partial_{t}^{i-1} \mathcal{L}^{\varepsilon}\right)^{\alpha_{i-1}} U^{\varepsilon}(\cdot)\left(\left(\varepsilon \partial_{t}\right)^{\beta} f\right),
$$

with $\alpha_{0}+2 \alpha_{1}+\cdots+i \alpha_{i-1}+\beta=i$, so that (29) is a direct consequence of Assumption 1 .

From the definition of $\mathcal{G}^{\varepsilon}$, one has, for all $0 \leq i+j \leq p+2$,

$$
\varepsilon^{i} \mathcal{G}_{(j)}^{\varepsilon(i)}[\cdot, u]\left(v_{1}, \ldots, v_{j}\right)=\left(\varepsilon \partial_{t}\right)^{i}\left(U^{\varepsilon}(-\cdot) f(\cdot)\right),
$$

with $f(t)=\mathcal{F}_{(j)}^{\varepsilon}\left[t, U^{\varepsilon}(t) u\right]\left(U^{\varepsilon}(t) v_{1}, \ldots, U^{\varepsilon}(t) v_{j}\right)$ so that one deduces directly from (29) that for all $s \geq s_{0}+i m$,

$$
\left|\left(\varepsilon \partial_{t}\right)^{i} \mathcal{G}_{(j)}^{\varepsilon}[\cdot, u]\left(v_{1}, \ldots, v_{j}\right)\right|_{X_{T}^{s-i m}} \leq C(s, T)|f|_{X_{(i)}^{s}} .
$$

Writing $\underline{u}:=U^{\varepsilon}(\cdot) u$ and $\underline{v}_{q}:=U^{\varepsilon}(\cdot) v_{q}$, one has $f=\mathcal{F}_{(j)}^{\varepsilon}[t, \underline{u}]\left(\underline{v}_{1}, \ldots, \underline{v}_{j}\right)$ and for all $0 \leq l \leq i,\left(\varepsilon \partial_{t}\right)^{l} f$ is a sum of terms of the form

$$
\varepsilon^{l_{0}} \mathcal{F}_{\left(j+\gamma_{1}+\cdots+\gamma_{l}\right)}^{\varepsilon\left(l_{0}\right)}[t, \underline{u}]\left(\left(\varepsilon \partial_{t}\right)^{l_{1}} \underline{v}_{1}, \ldots,\left(\varepsilon \partial_{t}\right)^{l_{j}} \underline{v}_{j},\left[\varepsilon \partial_{t} \underline{u}\right]^{\gamma_{1}}, \ldots,\left[\left(\varepsilon \partial_{t}\right)^{l} \underline{u}^{\gamma_{l}}\right),\right.
$$

with $l_{0}+l_{1}+\cdots+l_{j}+\gamma_{1}+2 \gamma_{2}+\cdots+l \gamma_{l}=l$, and where $\left[\left(\varepsilon \partial_{t}\right)^{l} \underline{u}\right]^{\gamma_{l}}$ stands for the $\gamma_{l}$-uplet with $\left(\varepsilon \partial_{t}\right)^{l} \underline{u}$ on each component. It follows therefore from Assumption 2 that

$$
\begin{aligned}
& \left|\left(\varepsilon \partial_{t}\right)^{l} f\right|_{X_{T}^{s-l m}} \leq C\left(s, T,|\underline{u}|_{X_{(l)}^{s_{0}+(l+1) m}}\right) \\
& \quad \times\left(\sum_{q=1}^{j}\left|\underline{v}_{q}\right|_{X_{(l)}^{s+m}} \prod_{q^{\prime} \neq q}\left|\underline{v}_{q^{\prime}}\right|_{X_{(l)}^{s_{0}+(l+1) m}}+|\underline{u}|_{X_{(l)}^{s+m}} \prod_{q=1}^{j}\left|\underline{v}_{q}\right|_{X_{(l)}^{s_{0}+(l+1) m}}\right)
\end{aligned}
$$


and since (29) implies that for all $r \geq s_{0}+l m,|\underline{u}|_{X_{(l)}^{r}} \leq C(r, T)|u|_{X_{T}^{r}}=C(r, T)|u|_{r}$ and $\left|\underline{v}_{q}\right|_{X_{(l)}^{r}} \leq C(r, T)\left|v_{q}\right|_{X_{T}^{r}}=C(r, T)\left|v_{q}\right|_{r}(q=1, \ldots, j)$, one deduces

$$
\begin{aligned}
\forall 0 \leq & l \leq i, \quad\left|\left(\varepsilon \partial_{t}\right)^{l} f\right|_{X_{T}^{s-l m}} \leq C\left(s, T,|u|_{\left.s_{0}+(l+1) m\right)}\right. \\
& \times\left(\sum_{q=1}^{j}\left|v_{q}\right|_{s+m} \prod_{q^{\prime} \neq q}\left|v_{q^{\prime}}\right|_{s_{0}+(l+1) m}+|u|_{s+m} \prod_{q=1}^{j}\left|v_{q}\right|_{s_{0}+(l+1) m}\right) .
\end{aligned}
$$

Together with (30), this shows that $\mathcal{G}^{\varepsilon}$ satisfies Assumption 2 .

Lemma 2 can then be generalized as follows:

Lemma 2'. Suppose that Assumptions [1' '?' are satisfied, and let $s \geq s_{0}+(p+1) m$. Assume also that $u_{k}^{\varepsilon} \in E_{(p+1)}^{s+d_{1}}$ and $\Phi^{\varepsilon}\left(u_{k}^{\varepsilon}\right) \in F_{(p)}^{s+d_{1}^{\prime}}$.

Then there exists a unique solution $v_{k}^{\varepsilon} \in E_{(p+1)}^{s}$ to (9) and

$\left|v_{k}^{\varepsilon}\right|_{E_{(p+1)}^{s}} \leq C\left(\varepsilon_{0}, s, T,\left|u_{k}^{\varepsilon}\right|_{\left.E_{(p+1)}^{s_{0}+(p+1) m+d_{1}}\right)}\right)\left(\left|\Phi^{\varepsilon}\left(u_{k}^{\varepsilon}\right)\right|_{F_{(p)}^{s+d_{1}^{\prime}}}+\left|\Phi^{\varepsilon}\left(u_{k}^{\varepsilon}\right)\right|_{F_{(p)}^{s_{0}+m+d_{1}^{\prime}}}\left|u_{k}^{\varepsilon}\right|_{E_{(p+1)}^{s+d_{1}}}\right)$.

Proof. Following the same steps as in the proof of Lemma 2, one can prove that $\left|v_{k}^{\varepsilon}\right|_{E_{(1)}^{s}}$ is bounded from above by the r.h.s. of the estimate given in the statement of the lemma. The lemma thus follows by finite induction: we just have to prove that the desired estimate on $v_{k}^{\varepsilon}$ holds in $E_{(l+1)}^{s}(1 \leq l \leq p)$ if it holds in $E_{\left(l^{\prime}+1\right)}^{s}$, for all $l^{\prime}<l$. Since moreover $\left|v_{k}^{\varepsilon}\right|_{E_{(l+1)}^{s}} \leq\left|v_{k}^{\varepsilon}\right|_{E_{(l)}^{s}}+\left|\left(\varepsilon \partial_{t}\right)^{l} \partial_{t} v_{k}^{\varepsilon}\right|_{X_{T}^{s-(l+1) m} \text {, we are reduced }}$ to prove that this latter term is bounded from above by the r.h.s. of the estimate given in the lemma. From the equation one gets

$$
\left|\left(\varepsilon \partial_{t}\right)^{l} \partial_{t} v_{k}^{\varepsilon}\right|_{X_{T}^{s-(l+1) m}} \leq\left|\left(\varepsilon \partial_{t}\right)^{l}\left(\mathcal{G}_{u}^{\varepsilon}\left[t, u_{k}^{\varepsilon}\right] v_{k}^{\varepsilon}\right)\right|_{X_{T}^{s-(l+1) m}}+\left|\left(\varepsilon \partial_{t}\right)^{l} \Phi_{1}\left(u_{k}^{\varepsilon}\right)\right|_{X_{T}^{s-(l+1) m}} .
$$

Proceeding exactly as for the obtention of (31) (with $j=1$ ) - but replacing $\mathcal{F}^{\varepsilon}$ by $\mathcal{G}^{\varepsilon}$ (which is possible thanks to Lemma 11), $\underline{u}$ by $u_{k}^{\varepsilon}$ and $\underline{v}_{1}$ by $v_{k}^{\varepsilon}$ - one gets

$\left|\left(\varepsilon \partial_{t}\right)^{l}\left(\mathcal{G}_{u}^{\varepsilon}\left[t, u_{k}^{\varepsilon}\right] v_{k}^{\varepsilon}\right)\right|_{X_{T}^{s-(l+1) m}} \leq C\left(s, T,\left|u_{k}^{\varepsilon}\right|_{X_{(l)}^{s_{0}+(l+1) m}}\right)\left(\left|v_{k}^{\varepsilon}\right|_{X_{(l)}^{s}}+\left|u_{k}^{\varepsilon}\right|_{X_{(l)}^{s}}\left|v_{k}^{\varepsilon}\right|_{X_{(l)}^{s_{0}+(l+1) m}}\right)$.

It follows therefore from (32) and (33) that $\left|\left(\varepsilon \partial_{t}\right)^{l} \partial_{t} v_{k}^{\varepsilon}\right|_{X_{T}^{s-(l+1) m}}$ is bounded from above by

$$
C\left(\varepsilon_{0}, s, T,\left|u_{k}^{\varepsilon}\right|_{E_{(l)}^{s_{0}+(l+1) m}}\right)\left(\left|v_{k}^{\varepsilon}\right|_{E_{(l)}^{s}}+\left|u_{k}^{\varepsilon}\right|_{E_{(l)}^{s}}\left|v_{k}^{\varepsilon}\right|_{E_{(l)}^{s_{0}+(l+1) m}}\right)+\left|\Phi_{1}\left(u_{k}^{\varepsilon}\right)\right|_{X_{(l)}^{s-m}} .
$$

and using the induction property thus gives the result.

Similarly, Lemmas 3 [5 must be replaced by the following generalizations to the case $p>0$; for Lemma 3, this is done in the following lemma.

Lemma 3'. Let $D \geq m+d_{1}^{\prime}$ and $s \geq s_{0}+m$. If, for some $M>0$, one has $\left|u_{j}^{\varepsilon}\right|_{E_{(p+1)}^{s+D}} \leq M(j=k, k+1)$, then

$$
\left|\Phi^{\varepsilon}\left(u_{k+1}^{\varepsilon}\right)\right|_{F_{(p)}^{s+d_{1}^{\prime}}} \leq C\left(\varepsilon_{0}, s, T, M\right)\left(\theta_{k}^{m+d_{1}^{\prime}-D}+\left|v_{k}^{\varepsilon}\right|_{E_{(p+1)}^{s+D}}\right)\left|v_{k}^{\varepsilon}\right|_{E_{(p+1)}^{s+D}} .
$$

Proof. One must add to the proof of Lemma 3 a control of $\left|\left(\varepsilon \partial_{t}\right)^{i} \phi_{1}\left(u_{k+1}^{\varepsilon}\right)\right|_{X_{T}^{s+d_{1}^{\prime}-i m}}$ $(i \leq p)$. Owing to (18), we are reduced to control $\varepsilon^{i} \partial_{t}^{i} E_{1}$ and $\varepsilon^{i} \partial_{t}^{i} E_{2}$ in $X_{T}^{s+d_{1}^{\prime}-i m}$. 
From the explicit expression of $E_{1}$ given in (19) and since Lemma 11 allows us to use Assumption 2 with $\mathcal{F}^{\varepsilon}$ replaced by $\mathcal{G}^{\varepsilon}$, one gets

$$
\left|\left(\varepsilon \partial_{t}\right)^{i} E_{1}\right|_{X_{T}^{s+d_{1}^{\prime}-i m}} \leq C\left(\varepsilon_{0}, s, T, M\right)\left|v_{k}\right|_{E_{(i)}^{s+D}}^{2}
$$

similarly, one gets from (20) that

$$
\left|\left(\varepsilon \partial_{t}\right)^{i} E_{2}\right|_{X_{T}^{s+d_{1}^{\prime}-i m}} \leq C\left(\varepsilon_{0}, s, T, M\right) \theta_{k}^{m+d_{1}^{\prime}-D}\left|v_{k}^{\varepsilon}\right|_{E_{(i+1)}^{s+D}},
$$

and the lemma follows.

The generalization of Lemma 4 is straightforward thanks to Lemma 22:

Lemma 4'. Let $D \geq d_{1}$ and $s \geq s_{0}+(p+1) m$. If, for some $M>0$, one has $\left|u_{k+1}^{\varepsilon}\right|_{E_{(p+1)}^{s+D}} \leq M$, then

$$
\left|v_{k+1}^{\varepsilon}\right|_{E_{(p+1)}^{s}} \leq C\left(\varepsilon_{0}, s, T, M\right)\left|\Phi^{\varepsilon}\left(u_{k+1}^{\varepsilon}\right)\right|_{F_{(p)}^{s+d_{1}^{\prime}}} .
$$

Finally, Lemma 5 is generalized as follows:

Lemma 5'. Let $P \geq D \geq \delta$ and $s \geq s_{0}+(p+1) m$. If, for some $M>0$, one has $\left|u_{k}^{\varepsilon}\right|_{E_{(p+1)}^{s+D}} \leq M$ and $\left|\left(h^{\varepsilon}, \underline{u}_{0}^{\varepsilon}\right)\right|_{F_{(p)}^{s+P-m}} \leq M$, then,

$$
\left|u_{k+1}^{\varepsilon}\right|_{E_{(p+1)}^{s+P}} \leq C\left(\varepsilon_{0}, s, T, M\right)\left(1+\theta_{k}^{\delta}\right)\left(1+\left|u_{k}^{\varepsilon}\right|_{E_{(p+1)}^{s+P}}\right) .
$$

If moreover $\left|u_{k+1}^{\varepsilon}\right|_{E_{(p+1)}^{s+D}} \leq M$, then one also has

$$
\left|v_{k+1}^{\varepsilon}\right|_{E_{(p+1)}^{s+P-\delta}} \leq C\left(\varepsilon_{0}, s, T, M\right)\left(1+\left|u_{k+1}^{\varepsilon}\right|_{E_{(p+1)}^{s+P}}\right) .
$$

Proof. Thanks to Lemma 2 one can generalize (26) for all $r \geq s_{0}+p m$ as

$$
\left|\Phi^{\varepsilon}(u)\right|_{F_{(p)}^{r}} \leq C\left(|u|_{\left.E_{(p+1)}^{s_{0}+m}\right)}\right)|u|_{E_{(p+1)}^{r+m}}+\operatorname{Cst}\left|\left(h^{\varepsilon}, \underline{u}_{0}^{\varepsilon}\right)\right|_{F_{(p)}^{r}}
$$

while (27) can be straightforwardly replaced by

$$
\left|u_{k+1}^{\varepsilon}\right|_{E_{(p+1)}^{s+P}} \leq\left|u_{k}^{\varepsilon}\right|_{E_{(p+1)}^{s+P}}+\operatorname{Cst} \theta_{k}^{\delta}\left|v_{k}^{\varepsilon}\right|_{E_{(p+1)}^{s+P-\delta}}
$$

Using Lemma 22 instead of Lemma 2, one concludes as in the proof of Lemma 5 .

The rest of the proof of the theorem is similar to the proof of Theorem 1

\subsection{A few remarks.}

3.2.1. Dependence on other parameters. The mappings $\mathcal{L}^{\varepsilon}$ and $\mathcal{F}^{\varepsilon}$ which appear in the IVP (1) may also depend on other parameters than $\varepsilon$. Theorems 1 (or $\mathbb{1}$ ) still hold, with an existence time independent of all these parameters as soon as all the constants which appear in Assumptions 1 13 (or [1-3i) are uniform with respect to these parameters (see Remark 7 below for such an example). 
3.2.2. Restricting the range of the assumptions. It sometimes occurs that Assumptions 2 (resp. 3 ) does not hold for all $u \in X^{s+m}$ (resp. $u \in X_{(p+1)}^{s+d_{1}}$ ) but only for $u \in \Omega_{0}$, with $\Omega_{0}$ an open subset of $X^{s+D}$ (resp. $X_{(p+1)}^{s+D}$ ). If for all $\theta_{0}$ one can find $u_{0}^{\varepsilon} \in \Omega_{0}$ such that conditions $(\mathrm{i})_{0}$, (ii) $)_{0}$ and (iii) $)_{0}$ of the induction proof of Theorems 1 and 1 are satisfied, then these theorems remain true. Indeed, by choosing $\theta_{0}$ large enough, one can make $\left|v_{k}^{\varepsilon}\right|_{E_{(p+1)}^{s+D}}(k \in \mathbb{N})$ small enough to have $u_{k}^{\varepsilon}:=u_{0}^{\varepsilon}+\sum_{l=0}^{k-1} S_{l} v_{l}^{\varepsilon} \in \Omega_{0}$.

In particular, the theorems still hold if the $u_{0}^{\varepsilon}$ provided by Lemma 7 belongs to $\Omega_{0}$.

Example 1. For the Serre and Green-Naghdi equations below, such restrictions on the range of validity of Assumptions 2 and 3 are imposed by the "nonzero depth condition" (36). The comment above shows that these restrictions are without consequence provided that (36) is initially satisfied.

3.2.3. Approximate linearization. The linear initial value problem (6) considered in Assumption 3 is the exact linearization of (11). One could replace it by an approximate linearization in the following sense (using the same notations as in Theorem [1] and with $\left.\mathcal{R}[t, u]:=\partial_{t} u+\frac{1}{\varepsilon} \mathcal{L}^{\varepsilon}(t) u+\mathcal{F}^{\varepsilon}[t, u]-h^{\varepsilon}\right)$ :

Proposition 1. Let $\mathbf{L} \in C\left([0, T] \times X^{s+D} ; \mathfrak{L}\left(X^{s+m}, X^{s}\right)\right)\left(s \geq s_{0}+m\right)$ be such that for all $t \in[0, T]$ and $u, v \in X^{s+D}$, one has

$$
\left|\mathcal{F}_{u}^{\varepsilon}[t, u] v-\mathbf{L}[t, u] v\right|_{X_{T}^{s+d_{1}^{\prime}}} \leq C\left(s, T,|u|_{s+D}\right)|\mathcal{R}[t, u]|_{X_{T}^{s+D}}|v|_{X_{T}^{s+D}}
$$

Then Theorem 1 still holds if the IVP (6) is replaced in Assumption 3 by

$$
\partial_{t} v^{\varepsilon}+\frac{1}{\varepsilon} \mathcal{L}^{\varepsilon}(t) v^{\varepsilon}+\mathbf{L}\left[t, u^{\varepsilon}\right] v^{\varepsilon}=f^{\varepsilon}, \quad v_{\left.\right|_{t=0}}^{\varepsilon}=g^{\varepsilon} .
$$

Remark 4. In other words, the proposition states that one can replace the derivative of $\mathcal{F}^{\varepsilon}$ in Assumption 3 by another linear operator, provided that the difference between both operators vanishes on the set of solutions of (1). This trick can sometimes simplify the computations (see for instance [8] and Remark 9 below).

Proof. In the proof of Theorem 1, we only use once the fact that the IVP (6) is the exact linearization of (1): in the derivation of (19) (otherwise, we only use the estimate provided by Assumption 10. Replacing $\mathcal{F}_{u}^{\varepsilon}\left[t, u^{\varepsilon}\right]$ by $\mathbf{L}\left[t, u^{\varepsilon}\right]$ in Assumption 3 thus implies the following modification of (18):

$$
\Phi_{1}\left(u_{k+1}^{\varepsilon}\right)=E_{1}+E_{2}+E_{3},
$$

where $E_{1}$ and $E_{2}$ are the same as in (18), while $E_{3}$ is given by

$$
E_{3}:=\mathcal{G}_{u}^{\varepsilon}\left[\cdot, u_{k}^{\varepsilon}\right] v_{k}^{\varepsilon}-\mathbf{G}\left[\cdot, u_{k}^{\varepsilon}\right] v_{k}^{\varepsilon}, \quad \text { with } \quad \mathbf{G}\left[\cdot, u_{k}^{\varepsilon}\right]:=U^{\varepsilon}(-t) \mathbf{L}\left[\cdot, U^{\varepsilon}(t) u_{k}^{\varepsilon}\right] .
$$

From Assumption 1 and the assumption made on $\mathbf{L}$, one gets

$$
\left|E_{3}\right|_{X_{T}^{s+d_{1}^{\prime}}} \leq C(s, T, M)\left|\Phi_{1}\left(u_{k}^{\varepsilon}\right)\right|_{X_{T}^{s+D}}\left|v_{k}^{\varepsilon}\right|_{X_{T}^{s+D}},
$$

where $M$ is as in Lemma 3. Therefore, the result given in Lemma 3 remains valid provided that $\left|\Phi_{1}\left(u_{k}^{\varepsilon}\right)\right|_{F^{s+D}} \leq C(s, T, M) \theta_{k}^{-q}$, with $q=D-m-d_{1}^{\prime}$. This point can be added without difficulty to the induction proof of Theorem 1

- The property is true for $k=0$ if $T$ is small enough (Lemma 7);

- If the property is true for some $k \in \mathbb{N}$, then we just saw that Lemma 3 remains true, so that $\left|\Phi_{1}\left(u_{k}^{\varepsilon}\right)\right|_{X_{T}^{s+d_{1}^{\prime}}} \leq C(s, T, M) \theta_{k}^{-2 q}=C(s, T, m) \theta_{k+1}^{-2 q / r}$ 
- We also get that $\left|\Phi_{1}\left(u_{k+1}^{\varepsilon}\right)\right|_{X_{T}^{s+P-m}} \leq C(s, T, M) \theta_{k+1}^{\alpha}$ (from (i) $\left.)_{k+1}\right)$;

- The estimate $\left|\Phi_{1}\left(u_{k+1}^{\varepsilon}\right)\right|_{X_{T}^{s+D}} \leq C(s, T, M) \theta_{k+1}^{-q}$ is then recovered by interpolation between the two estimates above.

It follows therefore that the proof is not affected by replacing $\mathcal{F}_{u}^{\varepsilon}$ by its approximation $\mathbf{L}$ in (6), which proves the proposition.

3.3. A stability property. We prove here a stability property for the IVP (1) which is very useful for the justification of asymptotic approximations of the exact solution. More precisely, assume that there exists an approximate solution $u_{a p p}^{\varepsilon}$ to (11) in the sense that

$$
\left\{\begin{array}{l}
\partial_{t} u_{\text {app }}^{\varepsilon}+\frac{1}{\varepsilon} \mathcal{L}^{\varepsilon}(t) u_{a p p}^{\varepsilon}+\mathcal{F}^{\varepsilon}\left[t, u_{a p p}^{\varepsilon}\right]=h^{\varepsilon}+\iota_{\varepsilon} R^{\varepsilon} \\
u_{a p p \mid t=0}^{\varepsilon}=\underline{u}_{0}^{\varepsilon}+\iota_{\varepsilon} r^{\varepsilon}
\end{array}\right.
$$

with $\iota_{\varepsilon}>0$ and $\left(R^{\varepsilon}, r^{\varepsilon}\right)_{0<\varepsilon<\varepsilon_{0}}$ bounded in some appropriate space. Our goal here is to prove that there exists an exact solution $\underline{u}^{\varepsilon}$ to (1) and that the error made by the approximation, namely $\underline{u}^{\varepsilon}-u_{a p p}^{\varepsilon}$, remains "small". An application of the following corollary is given in Theorem 3 below.

Corollary 1. Let the assumptions of Theorem [1' be satisfied and $s \geq s_{0}+(p+1) m$. Assume moreover that $\left(u_{\text {app }}^{\varepsilon}\right)_{0<\varepsilon<\varepsilon_{0}}$ and $\left(R^{\varepsilon}, r^{\varepsilon}\right)_{0<\varepsilon<\varepsilon_{0}}$ are bounded in $X_{(p)}^{s+P}$ and $F_{(p)}^{s+P}$ respectively.

There exist $0<\underline{T} \leq T$ and a unique family $\left(\underline{u}^{\varepsilon}\right)_{0<\varepsilon<\varepsilon_{0}}$ bounded in $C\left([0, \underline{T}] ; X^{s+D}\right)$ and solving the IVPs (11) $0<\varepsilon<\varepsilon_{0}$. Moreover, one has

$$
\left|\underline{u}^{\varepsilon}-u_{\text {app }}^{\varepsilon}\right|_{X_{T}^{s+D}} \leq \operatorname{Cst} \iota_{\varepsilon},
$$

and one can take $\underline{T}=T$ if $\iota_{\varepsilon}$ is small enough.

Proof. Let us seek an exact solution $u^{\varepsilon}$ under the form $u^{\varepsilon}=u_{a p p}^{\varepsilon}+\iota_{\varepsilon} e^{\varepsilon}$, which is equivalent to solving the IVP

$$
\left\{\begin{array}{l}
\partial_{t} e^{\varepsilon}+\frac{1}{\varepsilon} \mathcal{L}^{\varepsilon}(t) e^{\varepsilon}+\underline{\mathcal{F}}^{\varepsilon}\left[t, e^{\varepsilon}\right]=-R^{\varepsilon} \\
e_{\left.\right|_{t=0} ^{\varepsilon}}=-r^{\varepsilon}
\end{array}\right.
$$

with

$$
\underline{\mathcal{F}^{\varepsilon}}[t, u]:=\iota_{\varepsilon}^{-1}\left(\mathcal{F}^{\varepsilon}\left[t, u_{\text {app }}^{\varepsilon}+\iota_{\varepsilon} u\right]-\mathcal{F}^{\varepsilon}\left[t, u_{\text {app }}^{\varepsilon}\right]\right) .
$$

Lemma 8. The mapping $\underline{\mathcal{F}^{\varepsilon}}$ satisfies Assumption 团' for all s such that the family

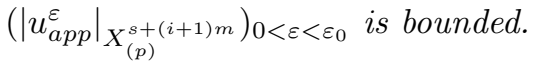

Proof. Let us first prove that $\underline{\mathcal{F}}^{\varepsilon}$ satisfies the estimates given in Assumption 2 when $j=0$. For all $0 \leq i \leq p$, one computes that $\varepsilon^{i} \underline{\mathcal{F}}^{\varepsilon(i)}[t, u]$ is a sum of terms of the form

$$
\iota_{\varepsilon}^{-1}\left(\varepsilon^{k} \mathcal{F}_{\left(j^{\prime}\right)}^{\varepsilon(k)}\left[t, u_{a p p}^{\varepsilon}+\iota_{\varepsilon} u\right]-\varepsilon^{k} \mathcal{F}_{\left(j^{\prime}\right)}^{\varepsilon(k)}\left[t, u_{a p p}^{\varepsilon}\right]\right)\left(\left[\left(\varepsilon \partial_{t}\right) u_{a p p}^{\varepsilon}\right]^{\alpha_{1}}, \ldots,\left[\left(\varepsilon \partial_{t}\right)^{i} u_{a p p}^{\varepsilon}\right]^{\alpha_{i}}\right),
$$

with $k+\alpha_{1}+\cdots+i \alpha_{i}=i$ and $j^{\prime}=\alpha_{1}+\cdots+\alpha_{i}$, so that we can use Assumption 2] to get

$$
\sup _{t \in[0, T]}\left|\varepsilon^{i} \underline{\mathcal{F}}^{\varepsilon(i)}[t, u]\right|_{s-i m} \leq C\left(s, t,\left|u_{a p p}^{\varepsilon}\right|_{X_{(p)}^{s+(i+1) m}}\right)|u|_{s+m}
$$


which proves the case $j=0$ since we assumed that $\left(\left|u_{a p p}^{\varepsilon}\right|_{\left.X_{(p)}^{s+(i+1) m}\right)_{0<\varepsilon<\varepsilon_{0}}}\right.$ is bounded.

Since for all $j \geq 1$ one has

$$
\underline{\mathcal{F}}_{(j)}^{\varepsilon}[t, u]\left(v_{1}, \ldots, v_{j}\right)=\iota_{\varepsilon}^{(j-1)} \mathcal{F}_{(j)}^{\varepsilon}\left[t, u_{a p p}^{\varepsilon}+\iota_{\varepsilon} u\right]\left(v_{1}, \ldots, v_{j}\right),
$$

the case $j \geq 1$ of the Assumption follows easily.

Thanks to the lemma and to the assumptions made in the statement of the corollary, one can use Theorem $\mathbb{1}$ with $\mathcal{F}^{\varepsilon}$ replaced by $\underline{\mathcal{F}}^{\varepsilon}$, and the first part of the corollary is proved.

We now prove that it is possible to take $T=\underline{T}$ when $\iota_{\varepsilon}$ is small enough. Instead of taking the first iterate $u_{0}^{\varepsilon}$ of the converging sequence $\left(u_{k}^{\varepsilon}\right)_{k}$ as given by Lemma 7. we can take $u_{0}^{\varepsilon}=u_{a p p}^{\varepsilon}$. Instead of shrinking $T$ to prove the first step of the induction as in the proof of Theorem 1 one must restrict to $\iota_{\varepsilon}$ small enough. This shows that an exact solution to (11) exists over $[0, T]$. In order to prove the error estimate, one proceeds as for the uniqueness part of Theorem 1 .

\section{Application to the Green-Naghdi and Serre equations}

This section is devoted to the proof of a well-posedness and stability result for the Green-Naghdi and Serre equations, which are among the most commonly used models in coastal oceanography.

4.1. The equations. The Green-Naghdi and Serre equations describe the motion of a layer of incompressible and irrotational fluid under the influence of gravity and under some assumptions on the physical regime considered. Defining the dimensionless parameters $\mu$ and $\varepsilon$ as

$$
\sqrt{\mu}:=\frac{\text { mean depth }}{\text { typical wave-length }} \quad \text { and } \quad \varepsilon:=\frac{\text { surface and bottom variations }}{\text { mean depth }},
$$

the Green-Naghdi and Serre regimes can be characterized as follows:

- Green-Naghdi regime: $\mu \ll 1$ and $\varepsilon \sim 1$;

- Serre regime: $\mu \ll 1$ and $\varepsilon \sim \sqrt{\mu}$.

A rigorous derivation of the Green-Naghdi and Serre models is performed in [2, to which we refer for more details. In nondimensionalized variables, the surface is parameterized at time $t$ by $\zeta(t, X)\left(X \in \mathbb{R}^{2}\right)$, while the bottom is parameterized by $b(X)$. Denoting by $V(t, X) \in \mathbb{R}^{2}$ the vertically averaged horizontal component of the velocity field at time $t$, the equations read (with $\varepsilon=1$ for the Green-Naghdi equations and $\varepsilon=\sqrt{\mu}$ for the Serre equations):

$$
\left\{\begin{array}{c}
(h+\mu \mathcal{T}[h, \varepsilon b]) \partial_{t} V+h \nabla \zeta+h \varepsilon(V \cdot \nabla) V \\
+\mu \varepsilon\left[\frac{1}{3} \nabla\left(h^{3} \mathcal{D}_{V} \operatorname{div}(V)\right)+\mathcal{Q}[h, \varepsilon b](V)\right]=0 \\
\partial_{t} \zeta+\nabla \cdot(h V)=0,
\end{array}\right.
$$

where $h:=1+\varepsilon(\zeta-b)$ while the linear operators $\mathcal{T}[h, b]$ and $\mathcal{D}_{V}$ and the quadratic form $\mathcal{Q}[h, b](\cdot)$ are defined as

$$
\begin{aligned}
\mathcal{T}[h, b] V & :=-\frac{1}{3} \nabla\left(h^{3} \nabla \cdot V\right)+\frac{1}{2}\left[\nabla\left(h^{2} \nabla b \cdot V\right)-h^{2} \nabla b \nabla \cdot V\right]+h \nabla b \nabla b \cdot V, \\
\mathcal{D}_{V} & :=-(V \cdot \nabla)+\operatorname{div}(V) \\
\mathcal{Q}[h, b](V) & :=\frac{1}{2} \nabla\left(h^{2}(V \cdot \nabla)^{2} b\right)+h\left(\frac{h}{2} \mathcal{D}_{V} \operatorname{div}(V)+(V \cdot \nabla)^{2} b\right) \nabla b .
\end{aligned}
$$


4.2. Well-posedness of the Serre and Green-Naghdi equations. Under the "nonzero depth condition"

$$
\exists h_{0}>0, \quad \inf _{\mathbb{R}^{2}} h \geq h_{0}, \quad(h=1+\varepsilon(\zeta-b))
$$

and after defining the spaces

$$
X^{s}:=\left\{(V, \zeta) \in H^{s}\left(\mathbb{R}^{2}\right)^{2} \times H^{s}\left(\mathbb{R}^{2}\right), \text { such that }|\nabla \cdot V|_{H^{s}}<\infty\right\},
$$

endowed with the norm

$$
|(V, \zeta)|_{X^{s}}:=\|V\|_{s}+|\zeta|_{H^{s}}, \quad \text { with } \quad\|V\|_{s}:=|V|_{H^{s}}+\sqrt{\mu}|\nabla \cdot V|_{H^{s}},
$$

one can prove the following well-posedness result on the Serre $(\varepsilon=\sqrt{\mu})$ and GreenNaghdi $(\varepsilon=1)$ equations:

Theorem 2 (Well-Posedness of the Serre and Green-Naghdi equations). Let $t_{0}>1$, $\varepsilon=\sqrt{\mu}$ (Serre) or $\varepsilon=1$ (Green-Naghdi), and $s \geq t_{0}+2$.

Let also $\left(V_{0}^{\mu}, \zeta_{0}^{\mu}\right)_{0<\mu<1}$ be bounded in $X^{s+38}$ and satisfy (36)).

Then there exists $T>0$ such that the Serre or Green-Naghdi equations (35) admit a unique family of solutions $\left(V^{\mu}, \zeta^{\mu}\right)_{0<\mu<1}$ bounded in $C\left(\left[0, \frac{T}{\varepsilon}\right] ; X^{s+4}\right)$ and with initial condition $\left(V_{0}, \zeta_{0}\right)_{0<\mu<1}$.

Remark 5. The spaces $X^{s+38}$ and $X^{s+4}$ correspond to the spaces $X^{s+P}$ and $X^{s+D}$ of Theorem 1 (one can check that $D=4, P=38$ is an admissible choice when $m=d_{1}=2$ and $\left.d_{1}^{\prime}=0\right)$.

Remark 6. For $1 D$ surface waves, flat bottoms $(b=0)$ and in the Green-Naghdi scaling $(\varepsilon=1)$, Y. A. Li [12] uses precise estimates on the inverse operator $(h+$ $\mu \mathcal{T}[h, \varepsilon b])^{-1}$ to obtain a well-posedness result in $H^{s+1}(\mathbb{R})^{2} \times H^{s}(\mathbb{R})$, with $s>3 / 2$ by a standard fixed point technique. It is not clear whether these techniques can be adapted to the $2 D H$ case: the identity $X^{s}=H^{s+1}\left(\mathbb{R}^{d}\right)^{d} \times H^{s}\left(\mathbb{R}^{d}\right)$ is false when $d=2$, and the smoothing properties of $(h+\mu \mathcal{T}[h, \varepsilon b])^{-1}$ can only be used to control the derivatives of $V$ which are in divergence form.

Proof. We only prove the theorem in the Serre scaling $(\varepsilon=\sqrt{\mu})$, which is the most difficult one because the existence time provided by the theorem is "large" (of order $O(1 / \varepsilon)$ ). The modifications to prove the theorem in the Green-Naghdi scaling are straightforward.

For the sake of simplicity, we write $\mathfrak{T}$ instead of $h+\mu \mathcal{T}[h, \varepsilon b]$ when no confusion is possible. It can be remarked that the operator $\mathfrak{T}$ is $L^{2}$ self-adjoint; since moreover, one has

$$
\begin{aligned}
& (h+\mu \mathcal{T}[h, \varepsilon b]) V, V)=(h V, V) \\
& \quad+\mu\left(h\left(\frac{h}{\sqrt{3}} \nabla \cdot V-\frac{\sqrt{3}}{2} \nabla b \cdot V\right), \frac{h}{\sqrt{3}} \nabla \cdot V-\frac{\sqrt{3}}{2} \nabla b \cdot V\right)+\frac{\mu}{4}(h \nabla b \cdot V, \nabla b \cdot V),
\end{aligned}
$$

and using the assumption that $\inf _{\mathbb{R}^{2}} h \geq h_{0}$, one deduces that

$$
(\mathfrak{T} V, V) \geq E[\varepsilon b](V)^{2},
$$

with $E[b](V)^{2}:=h_{0}|V|_{L^{2}}^{2}+\mu h_{0}\left|\frac{h}{\sqrt{3}} \nabla \cdot V-\frac{\sqrt{3}}{2} \nabla b \cdot V\right|_{L^{2}}^{2}+\mu \frac{h_{0}}{4}|\nabla b \cdot V|_{L^{2}}^{2}$.

It follows that $\mathfrak{T}$ has a self-adjoint, positive, inverse bounded on $L^{2}\left(\mathbb{R}^{2}\right)^{2}$ and the equations (35) can be recast under the form

$$
\partial_{t} u+\mathcal{L} u+\varepsilon \mathcal{F}^{\varepsilon}[u]=0,
$$




$$
\text { with } \begin{aligned}
u=(V, \zeta)^{T}, \mathcal{L}= & \left(\begin{array}{cc}
0 & \nabla \\
\operatorname{div} & 0
\end{array}\right), \mathcal{F}^{\varepsilon}[\cdot]=\left(\mathcal{F}_{1}^{\varepsilon}[\cdot], \mathcal{F}_{2}^{\varepsilon}[\cdot]\right)^{T} \text { and } \\
\mathcal{F}_{1}^{\varepsilon}[u] & =\frac{1}{\varepsilon}\left(\mathfrak{T}^{-1} h-1\right) \nabla \zeta+\mathfrak{T}^{-1} h(V \cdot \nabla) V \\
& +\mu \mathfrak{T}^{-1}\left[\frac{1}{3} \nabla\left(h^{3} \mathcal{D}_{V} \operatorname{div}(V)\right)+\mathcal{Q}[h, \varepsilon b](V)\right], \\
\mathcal{F}_{2}^{\varepsilon}[u] & =\nabla \cdot((\zeta-b) V) .
\end{aligned}
$$

The existence result stated by the theorem gives a time interval $\left[0, \frac{T}{\bar{\varepsilon}}\right]$ for (40). Rescaling time as $t \rightsquigarrow t / \varepsilon$, this is equivalent to solve the following equation on the time interval $[0, \underline{T}]$ :

$$
\partial_{t} u+\frac{1}{\varepsilon} \mathcal{L} u+\mathcal{F}^{\varepsilon}[u]=0
$$

this latter formulation is of the form (1), and the result thus follows from Theorem 1. provided that Assumptions [1]3 are satisfied. The rest of the proof is devoted to check that these assumptions are satisfied in the Banach scale $X^{s}$ defined in (37), with $s_{0}=t_{0}, m=d_{1}=2$, and $d_{1}^{\prime}=0$.

Remark 7. In the Green-Naghdi scaling $(\varepsilon=1)$, the parameter $\mu$ cannot be expressed in terms of $\varepsilon$. As seen in Section 3.2.1, in order for the theorem to be valid uniformly with respect to $\mu \in(0,1)$, we must check that Assumptions 1, 3] are satisfied uniformly in $\mu \in(0,1)$.

Remark 8. We always assume implicitly that the nonzero depth condition (36) is satisfied. As explained in Section 3.2.2, this is implied by the assumption that (36) is satisfied at $t=0$.

It follows immediately from the definition of $\mathcal{L}$ (independent of time here) that Assumption 1 is satisfied. In order to check the other assumptions, we need some preliminary results.

The next lemma gathers some general estimates; the first one is a classical Moser tame product estimate, the second one is a generalized Kato-Ponce commutator estimate (note that the estimate depends on $f$ only through its gradient, see Ths. 3 and 6 of [11]), and the last one is a classical "quasilinear type" estimate (e.g. Chapter II.C of [1]).

Lemma 9. Let $t_{0}>d / 2$ and $s \geq 0$.

i. For all $f, g \in H^{s} \cap H^{t_{0}}\left(\mathbb{R}^{d}\right)$, one has, using notation (2),

$$
|f g|_{H^{s}} \lesssim|f|_{H^{t_{0}}}|g|_{H^{s}}+\left\langle|f|_{H^{s}}|g|_{H^{t_{0}}}\right\rangle_{s>t_{0}}
$$

ii. Let $r \in \mathbb{R}$ be such that $-t_{0}<r \leq t_{0}+1$. Then, for all $f \in H^{t_{0}+1} \cap H^{s+r}\left(\mathbb{R}^{d}\right)$ and $u \in H^{t_{0}} \cap H^{s+r-1}\left(\mathbb{R}^{d}\right)$,

$$
\left|\left[\Lambda^{s}, f\right] u\right|_{H^{r}} \lesssim|\nabla f|_{H^{t_{0}}}|u|_{H^{s+r-1}}+\left\langle|\nabla f|_{H^{s+r-1}}|u|_{H^{t_{0}}}\right\rangle_{s>t_{0}+1-r} .
$$

iii. Let $N \in \mathbb{N}$, and $\mathbf{P}$ be a first order differential operator on $L^{2}\left(\mathbb{R}^{d}\right)^{N}$ with anti-adjoint principal part: $\mathbf{P}:=\sum_{j=1}^{d} P_{j}(x) \partial_{j}+P_{0}(x)$, with $P_{j}(x)$ symmetric for $j=1, \ldots, d$ and $x \in \mathbb{R}^{d}$. Then, for all $U \in H^{s} \cap H^{t_{0}+1}\left(\mathbb{R}^{d}\right)^{N}$,

$$
\left|\left(\Lambda^{s} \mathbf{P} U, \Lambda^{s} U\right)\right| \lesssim\left(\|\mathbf{P}\|_{H^{t_{0}+1}}|U|_{H^{s}}+\left\langle\|\mathbf{P}\|_{H^{s}}|U|_{H^{t_{0}+1}}\right\rangle_{s>t_{0}+1}\right)|U|_{H^{s}},
$$

with $\|\mathbf{P}\|_{H^{s}}:=\sum_{j=0}^{d}\left|P_{j}\right|_{H^{s}}$. 
The following lemma gives some properties on $\mathfrak{T}^{-1}$ which are necessary to check Assumption 2 (recall that the norm $\|\cdot\|_{s}$ has been defined in (38)).

Lemma 10. The following estimates hold for all $s \geq 0$ and uniformly with respect to $\mu \in(0,1)$ (and $\varepsilon=1$ or $\varepsilon=\sqrt{\mu})$ :

i. $\left\|\mathfrak{T}^{-1} V\right\|_{s} \leq c_{0}\left(|V|_{H^{s}}+\left\langle\left(|h|_{H^{s}}+|\nabla b|_{H^{s}}\right)|V|_{H^{t_{0}}}\right\rangle_{s>t_{0}+1}\right)$;

ii. $\sqrt{\mu}\left|\mathfrak{T}^{-1} \nabla \zeta\right|_{H^{s}} \leq c_{0}\left(|\zeta|_{H^{s}}+\left\langle\left(|h|_{H^{s}}+|\nabla b|_{H^{s}}\right)|\zeta|_{H^{t_{0}+1}}\right\rangle_{s>t_{0}+1}\right)$;

iii. $\frac{1}{\varepsilon}\left|\left(\mathfrak{T}^{-1} h-1\right) V\right|_{H^{s}} \leq c_{0}\left(|V|_{H^{s+1}}+\left\langle\left(|h|_{H^{s+1}}+|\nabla b|_{H^{s+1}}\right)|V|_{H^{t_{0}+1}}\right\rangle_{s>t_{0}}\right)$,

where $c_{0}$ is a constant depending only on $\frac{1}{h_{0}},|h|_{H^{t_{0}+1}}$ and $|\nabla b|_{H^{t_{0}+1}}$.

Proof. i. Remark first that

$$
|V|_{L^{2}}^{2} \leq \frac{1}{h_{0}} E[\varepsilon b](V)^{2} \quad \text { and } \quad \mu|\nabla \cdot V|_{L^{2}}^{2} \leq C\left(\frac{1}{h_{0}},|\nabla b|_{L^{\infty}}\right) E[\varepsilon b](V)^{2}
$$

replacing $V$ by $\mathfrak{T}^{-1} V$ in the above expressions and using (39) shows that

$$
\left|\mathfrak{T}^{-1} V\right|_{L^{2}}^{2} \leq \frac{1}{h_{0}}\left(V, \mathfrak{T}^{-1} V\right) \quad \text { and } \quad \mu\left|\nabla \cdot \mathfrak{T}^{-1} V\right|_{L^{2}}^{2} \leq C\left(\frac{1}{h_{0}},|\nabla b|_{L^{\infty}}\right)\left(V, \mathfrak{T}^{-1} V\right) .
$$

A simple Cauchy-Schwartz inequality thus yields

$$
\left\{\begin{array}{l}
\left\|\mathfrak{T}^{-1}\right\|_{L^{2}\left(\mathbb{R}^{2}\right)^{2} \rightarrow L^{2}\left(\mathbb{R}^{2}\right)^{2}} \leq \frac{1}{h_{0}} \\
\sqrt{\mu}\left\|\nabla \cdot \mathfrak{T}^{-1}\right\|_{L^{2}\left(\mathbb{R}^{2}\right)^{2} \rightarrow L^{2}\left(\mathbb{R}^{2}\right)} \leq C\left(\frac{1}{h_{0}},|\nabla b|_{L^{\infty}}\right) .
\end{array}\right.
$$

Using the fact that $\mathfrak{T}^{-1}$ is self-adjoint, one has $\left(\mathfrak{T}^{-1} \nabla\right)^{*}=-\nabla \cdot \mathfrak{T}^{-1}$, and thus

$$
\sqrt{\mu}\left\|\mathfrak{T}^{-1} \nabla\right\|_{L^{2}\left(\mathbb{R}^{2}\right) \rightarrow L^{2}\left(\mathbb{R}^{2}\right)^{2}} \leq C\left(\frac{1}{h_{0}},|\nabla b|_{\left.L^{\infty}\right)} .\right.
$$

We can now prove the following inequality

$$
E[\varepsilon b]\left(\Lambda^{s} \mathfrak{T}^{-1} V\right) \leq c_{0}\left(|V|_{H^{s}}+\left\langle\left(|h|_{H^{s}}+|\nabla b|_{H^{s}}\right)|V|_{H^{t_{0}}}\right\rangle_{s>t_{0}+1}\right),
$$

which, together with (42), obviously implies the first point of the lemma. From (39), one gets the relation

$$
\begin{gathered}
E[\varepsilon b]\left(\Lambda^{s} \mathfrak{T}^{-1} V\right)^{2} \leq\left(\Lambda^{s} V, \Lambda^{s} \mathfrak{T}^{-1} V\right)+\left(\mathfrak{T}\left[\Lambda^{s}, \mathfrak{T}^{-1}\right] V, \Lambda^{s} \mathfrak{T}^{-1} V\right) \\
(46)=\left(\Lambda^{s} V, \Lambda^{s} \mathfrak{T}^{-1} V\right)-\left(\left[\Lambda^{s}, h\right] \mathfrak{T}^{-1} V, \Lambda^{s} \mathfrak{T}^{-1} V\right)-\mu\left(\left[\Lambda^{s}, \mathcal{T}\right] \mathfrak{T}^{-1} V, \Lambda^{s} \mathfrak{T}^{-1} V\right) .
\end{gathered}
$$

Replacing $\mathcal{T}$ by its expression and integrating by parts, one gets therefore

$$
\begin{aligned}
E[\varepsilon b] & \left(\Lambda^{s} \mathfrak{T}^{-1} V\right)^{2} \leq\left(\Lambda^{s} V, \Lambda^{s} \mathfrak{T}^{-1} V\right)-\left(\left[\Lambda^{s}, h\right] \mathfrak{T}^{-1} V, \Lambda^{s} \mathfrak{T}^{-1} V\right) \\
& -\frac{1}{3}\left(\left[\Lambda^{s}, h^{3}\right]\left(\sqrt{\mu} \nabla \cdot \mathfrak{T}^{-1} V\right), \Lambda^{s}\left(\sqrt{\mu} \nabla \cdot \mathfrak{T}^{-1} V\right)\right) \\
& -\frac{\sqrt{\mu}}{2}\left(\left[\Lambda^{s}, h^{2} \nabla b^{T}\right] \mathfrak{T}^{-1} V, \Lambda^{s}\left(\sqrt{\mu} \nabla \cdot \mathfrak{T}^{-1} V\right)\right) \\
& +\frac{\sqrt{\mu}}{2}\left(\left[\Lambda^{s}, h^{2} \nabla b\right]\left(\sqrt{\mu} \nabla \cdot \mathfrak{T}^{-1} V\right), \Lambda^{s} \mathfrak{T}^{-1} V\right)+\mu\left(\left[\Lambda^{s}, h \nabla b \nabla b^{T}\right] \mathfrak{T}^{-1} V, \Lambda^{s} \mathfrak{T}^{-1} V\right) .
\end{aligned}
$$

Applying Cauchy-Schwartz's inequality to every component of the r.h.s. of the above expression, and using Lemma 9, ii and (39), one gets directly

$$
\begin{aligned}
& E[\varepsilon b]\left(\Lambda^{s} \mathfrak{T}^{-1} V\right) \leq C\left(\frac{1}{h_{0}},|h|_{H^{t_{0}+1}},|\nabla b|_{H^{t_{0}+1}}\right) \\
& \quad \times \quad\left(|V|_{H^{s}}+E[\varepsilon b]\left(\Lambda^{s-1} \mathfrak{T}^{-1} V\right)+\left\langle\left(|h|_{H^{s}}+|\nabla b|_{H^{s}}\right) E[\varepsilon b]\left(\Lambda^{t_{0}} V\right)\right\rangle_{s>t_{0}+1}\right),
\end{aligned}
$$


from which one deduces (45).

ii. Remark that

$$
\begin{aligned}
\sqrt{\mu}\left|\Lambda^{s} \mathfrak{T}^{-1} \nabla \zeta\right|_{2} & \leq \sqrt{\mu}\left|\mathfrak{T}^{-1} \nabla \Lambda^{s} \zeta\right|_{2}+\sqrt{\mu}\left|\left[\Lambda^{s}, \mathfrak{T}^{-1}\right] \nabla \zeta\right|_{2} \\
& \leq C\left(\frac{1}{h_{0}},|\nabla b|_{L^{\infty}}\right)|\zeta|_{H^{s}}+\sqrt{\mu}\left|\left[\Lambda^{s}, \mathfrak{T}^{-1}\right] \nabla \zeta\right|_{2}
\end{aligned}
$$

where we used (43) to control the first term of the r.h.s. For the second term, remark that $\left[\Lambda^{s}, \mathfrak{T}^{-1}\right]=-\mathfrak{T}^{-1}\left[\Lambda^{s}, \mathfrak{T}\right] \mathfrak{T}^{-1}$, so that

$$
\begin{aligned}
{\left[\Lambda^{s}, \mathfrak{T}^{-1}\right]=} & -\mathfrak{T}^{-1}\left[\Lambda^{s}, h\right] \mathfrak{T}^{-1}+\frac{1}{3}\left(\sqrt{\mu} \mathfrak{T}^{-1} \nabla\right)\left[\Lambda^{s}, h^{3}\right]\left(\sqrt{\mu} \nabla \cdot \mathfrak{T}^{-1}\right) \\
& -\frac{\sqrt{\mu}}{2}\left(\sqrt{\mu} \mathfrak{T}^{-1} \nabla\right)\left[\Lambda^{s}, h^{2} \nabla b\right] \cdot \mathfrak{T}^{-1}+\frac{\sqrt{\mu}}{2} \mathfrak{T}^{-1}\left[\Lambda^{s}, \nabla b\right]\left(\sqrt{\mu} \nabla \cdot \mathfrak{T}^{-1}\right) \\
& -\mu \mathfrak{T}^{-1}\left[\Lambda^{s}, \nabla b \nabla b^{T}\right] \mathfrak{T}^{-1}
\end{aligned}
$$

It follows that $\left|\left[\Lambda^{s}, \mathfrak{T}^{-1}\right] \nabla \zeta\right|_{L^{2}}$ can be controlled using Lemma 9]ii, the estimates on $\left\|\mathfrak{T}^{-1}\right\|_{L^{2} \rightarrow L^{2}}$ and $\sqrt{\mu}\left\|\mathfrak{T}^{-1} \nabla\right\|_{L^{2} \rightarrow L^{2}}$ given by (43) and (44), and the estimate on $\left\|\mathfrak{T}^{-1}\right\|_{H^{s} \rightarrow H^{s}}$ and $\sqrt{\mu}\left\|\nabla \cdot \mathfrak{T}^{-1}\right\|_{H^{s} \rightarrow H^{s}}$ given by i. Together with (47), this proves ii.

iii. Just remark that $1-\mathfrak{T}^{-1} h=\mathfrak{T}^{-1}(\mathfrak{T}-h)=\mu \mathfrak{T}^{-1} \mathcal{T}$, so that

$$
\frac{1}{\varepsilon}\left|\left(1-\mathfrak{T}^{-1} h\right) V\right|_{H^{s}} \leq \frac{\sqrt{\mu}}{\varepsilon}\left|\sqrt{\mu} \mathfrak{T}^{-1} \mathcal{T} V\right|_{H^{s}}
$$

Since $\sqrt{\mu} / \varepsilon \leq 1$, the result follows from the first two points and Lemma $9 \mathrm{i}$.

A direct consequence of Lemmas $9 \mathrm{i}$ and $10 \mathrm{i}$ and .iii, and of the definition of $\mathcal{F}^{\varepsilon}$, is that the first part of Assumption 2 holds with $s=t_{0}$ and $m=2$.

Remarking that

$$
d_{\zeta}\left(\zeta \mapsto \mathfrak{T}^{-1}\right) \widetilde{\zeta}=-\mathfrak{T}^{-1}\left(\varepsilon \widetilde{\zeta}+\mu d_{\zeta}(\zeta \mapsto \mathcal{T}[h, \varepsilon b]) \widetilde{\zeta}\right) \mathfrak{T}^{-1}
$$

and using Lemmas 9, $\mathrm{i}$ and 10, $\mathrm{i}$ and .ii, one can check that the conditions on the first and second derivative of $\mathcal{F}^{\varepsilon}$ made in Assumption 2 are also satisfied.

In order to check Assumption 3 , we must study the Cauchy problem associated to the linearization of (41) around some reference state $\underline{u}:=(\underline{V}, \underline{\zeta})$ :

$$
\partial_{t} u+\frac{1}{\varepsilon} \mathcal{L} u+\mathcal{F}_{u}^{\varepsilon}[\underline{u}] u=f, \quad u_{\mid t=0}=g,
$$

with $f=\left(F_{1}, f_{2}\right)^{T}$ and $g:=\left(G_{1}, g_{2}\right)^{T}$.

From the explicit expression of $\mathcal{L}$ and $\mathcal{F}^{\varepsilon}$ given in (40), and writing $\underline{h}:=1+\varepsilon(\underline{\zeta}-b)$, $\underline{\mathcal{I}}:=\mathcal{T}[\underline{h}, \varepsilon b]$, and $\underline{\mathfrak{T}}:=\underline{h}+\mu \underline{\mathcal{I}}$, one gets that (48) is equivalent to

$$
\left(\begin{array}{c}
(\underline{h}+\mu \underline{\mathcal{T}}) \partial_{t} V \\
\partial_{t} \zeta
\end{array}\right)+\left(\begin{array}{cc}
\mathcal{N}_{1} & \mathcal{N}_{2} \\
\mathcal{N}_{3} & \mathcal{N}_{4}
\end{array}\right)\left(\begin{array}{c}
V \\
\zeta
\end{array}\right)=\left(\begin{array}{c}
(\underline{h}+\mu \underline{\mathcal{T}}) F_{1} \\
f_{2}
\end{array}\right)
$$


with initial condition $(V, \zeta)_{\mid t=0}=\left(G_{1}, g_{2}\right)$, and where the linear operators $\mathcal{N}_{j}(j=$ $1, \ldots, 4)$ are given by

$$
\begin{aligned}
\mathcal{N}_{1} V:= & \underline{h}(\underline{V} \cdot \nabla) V+\underline{h}(V \cdot \nabla) \underline{V}+\frac{\mu}{3} \nabla\left[\underline{h}^{3}\left(\mathcal{D}_{\underline{V}}(\nabla \cdot V)+\mathcal{D}_{V}(\nabla \cdot \underline{V})\right)\right], \\
& +\mu \mathcal{Q}[\underline{h}, \varepsilon b](V, \underline{V}), \\
\mathcal{N}_{2} \zeta:= & \frac{1}{\varepsilon} \underline{h} \nabla \zeta+\underline{\mathfrak{b}} \zeta+\mu \nabla(\underline{h \mathfrak{a}} \zeta), \\
\mathcal{N}_{3} V:= & \frac{1}{\varepsilon} \nabla \cdot(\underline{h} V), \\
\mathcal{N}_{4} \zeta:= & \nabla \cdot(\zeta \underline{V}),
\end{aligned}
$$

with

$$
\begin{aligned}
& \underline{\mathfrak{a}}:=\varepsilon \underline{h} \mathcal{D}_{\underline{V}}(\nabla \cdot \underline{V})+(\underline{V} \cdot \nabla)^{2} b-(\nabla b-\underline{h} \nabla) \cdot\left(\nabla \underline{\zeta}+\varepsilon \mathcal{F}_{1}^{\epsilon}[\underline{u}]\right), \\
& \underline{\mathfrak{b}}:=\varepsilon(\underline{V} \cdot \nabla) \underline{V}-\varepsilon \mathcal{F}_{1}^{\epsilon}[\underline{u}]+\mu \underline{\mathfrak{a}} \nabla b,
\end{aligned}
$$

and with $\mathcal{Q}[\underline{h}, \varepsilon b](\cdot, \cdot)$ standing for the bilinear symmetric form canonically associated to $\mathcal{Q}[\underline{h}, \varepsilon b]$.

Remark 9. Thanks to Proposition 1 one can replace $\nabla \underline{\zeta}+\varepsilon \mathcal{F}_{1}^{\varepsilon}[\underline{u}]$ by $-\varepsilon \partial_{t} \underline{V}$ in the definition of $\underline{\mathfrak{a}}$ and $-\varepsilon \mathcal{F}_{1}^{\varepsilon}[\underline{u}]$ by $\varepsilon \partial_{t} \underline{V}+\nabla \underline{\zeta}$ in the definition of $\underline{\mathfrak{b}}$. It follows that $\sqrt{\mu}|\underline{\mathfrak{a}}|_{H^{s}}$ and $|\underline{\mathfrak{b}}|_{H^{s}}$ are bounded from above by

$$
C\left(|\underline{\zeta}|_{H^{t_{0}}},\|\underline{V}\|_{t_{0}+1},|b|_{H^{t_{0}+1}},\left\|\varepsilon \partial_{t} \underline{\underline{V}}\right\|_{t_{0}}\right)\left(|\underline{\zeta}|_{H^{s}}+\|\underline{V}\|_{s+1}+\left\|\varepsilon \partial_{t} \underline{V}\right\|_{s}+|b|_{H^{s+1}}\right) .
$$

The following lemma end the proof of the theorem since it implies that Assumption 3 is also satisfied with $s_{0}=t_{0}, m=2, d_{1}=2$ and $d_{1}^{\prime}=0$ (recall that the notation $\mathcal{I}^{s}$ is defined in (5D):

Lemma 11. Let $T>0, f=\left(F_{1}, f_{2}\right) \in C\left([0, T] ; X^{s}\right), g \in X^{s}$ and $\underline{u}=(\underline{V}, \underline{\zeta}) \in$ $X_{T}^{s+1}$ be such that $\partial_{t} \underline{u} \in X_{T}^{s}$. Then for all $s \geq 0$, there exists a unique solution $u=(V, \zeta) \in X_{T}^{s}$ to 48) and for all $0 \leq t \leq T$,

$$
|u(t)|_{X^{s}} \leq \underline{c}_{1}\left(\mathcal{I}^{s}(t, f, g)+\left\langle\left(|\underline{u}|_{X_{T}^{s+1}}+\left|\varepsilon \partial_{t} \underline{u}\right|_{X_{T}^{s}}\right) \mathcal{I}^{t_{0}+1}(t, f, g)\right\rangle_{s>t_{0}+1}\right),
$$

with $\underline{c}_{1}=C\left(T, \frac{1}{h_{0}},|\underline{u}|_{X_{T}^{t_{0}+2}},\left.\left|\varepsilon \partial_{t} \underline{u}_{X_{T}^{t_{0}+1}},\right| b\right|_{H^{t_{0}+2}},|b|_{H^{s+2}}\right)$.

Proof. We only prove the energy estimate, since the existence/uniqueness of a solution to the linear Cauchy problem (49) can be classically obtained by regularization techniques. Multiplying the first equation of (49) by $\underline{\mathfrak{T}} \Lambda^{s} \underline{\mathfrak{T}}^{-1}$ and the second by $\Lambda^{s}$, and taking the scalar product with $\Lambda^{s} V$ and $\Lambda^{s} \zeta$ respectively, one gets

$$
\begin{aligned}
\frac{1}{2} \partial_{t} & \left(\underline{\mathfrak{T}} \Lambda^{s} V, \Lambda^{s} V\right)+\frac{1}{2} \partial_{t}\left(\Lambda^{s} \zeta, \Lambda^{s} \zeta\right)=-\left(\Lambda^{s} \mathcal{N}_{1} V, \Lambda^{s} V\right)-\left(\Lambda^{s} \mathcal{N}_{4} \zeta, \Lambda^{s} \zeta\right) \\
& -\left[\left(\Lambda^{s} \mathcal{N}_{2} \zeta, \Lambda^{s} V\right)+\left(\Lambda^{s} \mathcal{N}_{3} V, \Lambda^{s} \zeta\right)\right] \\
& +\left(\left[\Lambda^{s}, \mathfrak{T}\right] \underline{\mathfrak{T}}^{-1} \mathcal{N}_{1} V, \Lambda^{s} V\right)+\left(\left[\Lambda^{s}, \underline{\mathfrak{T}}\right] \underline{\mathfrak{T}}^{-1} \mathcal{N}_{2} \zeta, \Lambda^{s} V\right) \\
& +\left(\underline{\mathfrak{T}} \Lambda^{s} F_{1}, \Lambda^{s} V\right)+\left(\Lambda^{s} f_{2}, \Lambda^{s} \zeta\right)+\frac{1}{2}\left(\partial_{t} \underline{\mathfrak{T}} \Lambda^{s} V, \Lambda^{s} V\right)
\end{aligned}
$$

we now prove that (with $u=(V, \zeta), \underline{u}=(\underline{V}, \underline{\zeta}), f=\left(F_{1}, f_{2}\right)$, and $|\cdot|_{X^{s}}$ as defined in (38))

$$
\begin{aligned}
& \partial_{t}\left(\underline{\mathfrak{T}} \Lambda^{s} V, \Lambda^{s} V\right)+\partial_{t}\left(\Lambda^{s} \zeta, \Lambda^{s} \zeta\right) \\
& \quad \leq \underline{c}_{1}\left(|u|_{X^{s}}+|f|_{X^{s}}+\left\langle\left(|\underline{u}|_{X^{s+1}}+\left|\varepsilon \partial_{t} \underline{u}\right|_{X^{s}}\right)|u|_{X^{t_{0}+1}}\right\rangle_{s>t_{0}+1}\right)|u|_{X^{s}} .
\end{aligned}
$$


We thus check that all the components of the r.h.s. of (50) are bounded from above by the r.h.s. of (51).

- Control of $\left(\Lambda^{s} \mathcal{N}_{1} V, \Lambda^{s} V\right)$. Let us first rewrite

$$
\begin{aligned}
\left(\Lambda^{s} \mathcal{N}_{1} V, \Lambda^{s} V\right)= & -\frac{1}{2} \mu I+\left(\Lambda^{s} \mathbf{A} V, \Lambda^{s} V\right)+\mu\left(\Lambda^{s} \mathbf{B}(\nabla \cdot V), \Lambda^{s}(\nabla \cdot V)\right) \\
& +\mu\left(\Lambda^{s} \mathbf{C} V, \Lambda^{s}(\nabla \cdot V)\right)+\mu\left(\Lambda^{s} \mathbf{D}(\nabla \cdot V), \Lambda^{s} V\right)
\end{aligned}
$$

with

$$
\begin{aligned}
\mathbf{A} V:= & \underline{h}(\underline{\underline{\mu}} \cdot \nabla)(V+\mu(V \cdot \nabla b) \nabla b)+\underline{h}(V \cdot \nabla)(\underline{V}+\mu(\underline{V} \cdot \nabla b) \nabla b) \\
& -\frac{\underline{h}}{2} \underline{h}^{2}(V \cdot \nabla)(\nabla \cdot \underline{V}) \nabla b, \\
\mathbf{B}:= & -\frac{1}{3} \underline{h}^{3} \mathcal{D}_{\underline{V}}-\frac{1}{3} \underline{h}^{3}(\nabla \cdot \underline{V}), \\
\mathbf{C} V:= & \frac{1}{3} \underline{h}^{3}(V \cdot \nabla)(\nabla \cdot \underline{V})-\frac{1}{2} \underline{h}^{2}(V \cdot \nabla)(\underline{V} \cdot \nabla b), \\
& +\left[\underline{h}^{2}(\underline{V} \cdot \nabla)+\left(\underline{h}^{2}(\underline{V} \cdot \nabla)\right)^{*}\right] \nabla b \cdot V \\
\mathbf{D}:= & \underline{h}^{2} \nabla b(\nabla \cdot \underline{V}),
\end{aligned}
$$

and $I:=\left(\left[\Lambda^{s}, \underline{h}^{2} \nabla b(\underline{V} \cdot \nabla)\right] \nabla \cdot V, \Lambda^{s} V\right)+\left(\left[\Lambda^{s}, \underline{h}^{2}(\underline{V} \cdot \nabla)\left(\nabla b^{T} \cdot\right)\right] V, \Lambda^{s} \nabla \cdot V\right)$. One can check that $\mu I$ is bounded from above by the r.h.s. of (51) by applying CauchySchwartz's inequality to its two components, and then using (42) and Lemma 9, ii. Remarking also that $\mathbf{A}$ and $\mathbf{B}$ are first order differential operators with anti-adjoint principal part, one can use Lemma 9,iii and (42) to check that the second and third component of (52) are bounded from above by the r.h.s. of (51). Finally, we can prove that the same control holds on the last two components of (52) by using Cauchy-Schwartz's inequality and Lemma 9, i (remark that $\mathbf{C}$ and $\mathbf{D}$ are simple matrix and scalar valued functions).

- Control of $\left(\Lambda^{s} \mathcal{N}_{4} \zeta, \Lambda^{s} \zeta\right)$. From the explicit expression of $\mathcal{N}_{4}$ and Lemma 9, iii, one obtains directly that this term is controlled by the r.h.s. of (51).

- Control of $\left(\Lambda^{s} \mathcal{N}_{2} \zeta, \Lambda^{s} V\right)+\left(\Lambda^{s} \mathcal{N}_{3} V, \Lambda^{s} \zeta\right)$. Integrating by parts, one gets immediately that

$$
\begin{aligned}
\left(\Lambda^{s} \mathcal{N}_{2} \zeta, \Lambda^{s} V\right)+\left(\Lambda^{s} \mathcal{N}_{3} V, \Lambda^{s} \zeta\right)= & \left(\Lambda^{s}(\underline{\mathfrak{b}} \zeta), \Lambda^{s} V\right)-\left(\Lambda^{s}(\underline{h}(\sqrt{\mu} \underline{\mathfrak{a}}) \zeta), \sqrt{\mu} \Lambda^{s} \nabla \cdot V\right) \\
& +\frac{1}{\varepsilon}\left(\left[\Lambda^{s}, \underline{h}\right] \nabla \zeta, \Lambda^{s} V\right)+\frac{1}{\varepsilon}\left(\nabla\left[\Lambda^{s}, \underline{h}\right] V, \Lambda^{s} \zeta\right) .
\end{aligned}
$$

The first two components can be controlled by a Cauchy-Schwartz inequality and Lemma 9.i, and the last two by Cauchy-Schwartz and Lemma 9,ii (note that the commutator estimate provided by this lemma depends only on $\underline{h}$ through $\nabla \underline{h}=$ $\varepsilon \nabla(\zeta-b)$ and provides therefore the $\varepsilon$ necessary to compensate the singular term $1 / \varepsilon)$. Together with the estimates of Remark 9, this shows that this term also is controlled by the r.h.s. of (51).

- Control of $\left(\left[\Lambda^{s}, \underline{\mathfrak{T}}\right] \underline{\mathfrak{T}}^{-1} \mathcal{N}_{1} V, \Lambda^{s} V\right)$. Let us remark that $\mathcal{N}_{1} V$ can be written as

$$
\mathcal{N}_{1} V=\underline{\mathfrak{T}} H+\mathbf{P}_{1} V+\mathbf{P}_{2}(\sqrt{\mu} \nabla \cdot V)
$$

with $H:=\left[(\nabla \cdot V) \underline{V}-\frac{3}{2}(V \cdot \nabla b) \underline{V}\right]$, and where $\mathbf{P}_{1}$ and $\mathbf{P}_{2}$ are both first order differential operators, and whose coefficients are polynomial expressions of $\sqrt{\mu}, h$, $\nabla h, \sqrt{\mu} \nabla \cdot \underline{V}, \nabla(\sqrt{\mu} \nabla \cdot \underline{V})$ and of the vectors $\underline{V}$ and $\nabla b$ and their first derivatives 
(the exact expression of $\mathbf{P}_{1}$ and $\mathbf{P}_{2}$ is of no importance). One has therefore

$$
\begin{aligned}
{\left[\Lambda^{s}, \underline{\mathfrak{T}}\right] \underline{\mathfrak{T}}^{-1} \mathcal{N}_{1} V=} & {\left[\Lambda^{s}, \underline{\mathfrak{T}}\right] H+\left[\Lambda^{s}, \underline{\mathfrak{T}}\right] \underline{\mathfrak{T}}^{-1}\left(\mathbf{P}_{1} V+\mathbf{P}_{2}(\sqrt{\mu} \nabla \cdot V)\right) } \\
= & {\left[\Lambda^{s}, \underline{h}\right] H+\left[\Lambda^{s}, \underline{h}\right] \mathfrak{\mathfrak { T }}^{-1}\left(\mathbf{P}_{1} V+\mathbf{P}_{2}(\sqrt{\mu} \nabla \cdot V)\right) } \\
& +\mu\left[\Lambda^{s}, \mathcal{T}\right] H+\mu\left[\Lambda^{s}, \mathcal{T}\right] \underline{\mathfrak{T}}^{-1}\left(\mathbf{P}_{1} V+\mathbf{P}_{2}(\sqrt{\mu} \nabla \cdot V)\right) \\
:= & I_{1}+I_{2}+I_{3}+I_{4} .
\end{aligned}
$$

One deduces directly from Lemmas 9, ii and 10, i and the definition of $H, \mathbf{P}_{1}$ and $\mathbf{P}_{2}$ that

$$
\left|I_{1}\right|_{L^{2}}+\left|I_{2}\right|_{L^{2}} \leq \underline{c}_{1}\left(\|V\|_{s}+\left\langle\left(\|\underline{V}\|_{H^{s}}+|\underline{h}|_{H^{s}}+|\nabla b|_{H^{s}}\right)\|V\|_{t_{0}+1}\right\rangle_{s>t_{0}+1}\right),
$$

and a simple Cauchy-Schwartz inequality shows that scalar products $\left(I_{1}, \Lambda^{s} V\right)$ and $\left(I_{2}, \Lambda^{s} V\right)$ are controlled by the r.h.s. of (51). Proceeding exactly as for the control of the third term of (46), one can check that the same control holds for $\left(I_{3}, \Lambda^{s} V\right)$ and $\left(I_{4}, \Lambda^{s} V\right)$.

- Control of $\left(\left[\Lambda^{s}, \underline{\mathfrak{T}}\right] \underline{\mathfrak{T}}^{-1} \mathcal{N}_{2} \zeta, \Lambda^{s} V\right)$. Using the explicit expression of $\underline{\mathfrak{T}}$ and Lemma [10, and proceeding as for the control of the third term of (46), one can bound this term from above by the r.h.s. of (51).

- Control of the last three terms of (50). Controlling these terms by the r.h.s. of (51) follows directly from a Cauchy-Schwartz inequality (and an integration by parts for the $O(\mu)$ component of $\underline{\mathfrak{T}}$ and $\left.\partial_{t} \mathfrak{T}\right)$.

We can now conclude the proof of the lemma. Recalling that from (39), (42) and the definition (38), one has

$$
\begin{aligned}
\left(\underline{\mathfrak{T}} \Lambda^{s} V, \Lambda^{s} V\right)+\left(\Lambda^{s} \zeta, \Lambda^{s} \zeta\right) & \leq C\left(|\underline{h}|_{L^{\infty}},|\nabla b|_{L^{\infty}}\right)|u|_{X^{s}}^{2} \\
|u|_{X^{s}}^{2} & \leq C\left(\frac{1}{h_{0}},|\nabla b|_{L^{\infty}}\right)\left(\underline{\mathfrak{T}} \Lambda^{s} V, \Lambda^{s} V\right)+\left(\Lambda^{s} \zeta, \Lambda^{s} \zeta\right),
\end{aligned}
$$

one can integrate (51) with respect to time to obtain, for all $t \in[0, T]$,

$|u(t)|_{X^{s}} \leq \underline{c}_{1}\left[|u(0)|_{X^{s}}+\int_{0}^{t}\left(|u|_{X^{s}}+|f|_{X^{s}}+\left\langle\left(|\underline{u}|_{X^{s+1}}+\left|\varepsilon \partial_{t} \underline{u}\right|_{X^{s}}\right)|u|_{X^{t_{0}+1}}\right\rangle_{s>t_{0}+1}\right)\right]$.

Using this identity with $s=t_{0}+1$ shows that

$$
|u(t)|_{X^{t_{0}+1}} \leq \underline{c}_{1}\left[|u(0)|_{X^{t_{0}+1}}+\int_{0}^{t}\left(|u|_{X^{t_{0}+1}}+|f|_{X^{t_{0}+1}}\right)\right]
$$

and Gronwall's lemma thus yields, for all $t \in[0, T]$,

$$
|u(t)|_{X^{t_{0}+1}} \leq \underline{c}_{1}\left(|u(0)|_{X^{t_{0}+1}}+\int_{0}^{t} \sup _{0 \leq t^{\prime \prime} \leq t^{\prime}}\left|f\left(t^{\prime \prime}\right)\right|_{X^{t_{0}+1}} d t^{\prime}\right)
$$

(recall that $\underline{c}_{1}$ is a generic notation whose value can change from one line to another); plugging this expression into (54) thus ends the proof of the lemma.

4.3. Justification of the Serre and Green-Naghdi models. As said above, the Serre and Green-Naghdi are both asymptotic models which describe the dynamics of the water-waves equations. It is not known however whether these asymptotics are correct, in the sense that the exact solutions to the asymptotic models provide a correct approximation to the exact solutions of the water-waves equations. This is what we show below: if solutions $\left(V_{a p p}^{\mu}, \zeta_{a p p}^{\mu}\right)$ to the water-waves equations exist and 
approximately solve (35), then their asymptotic behavior (as $\mu \rightarrow 0$ ) is correctly described by the Serre $(\varepsilon=\sqrt{\mu})$ or Green-Naghdi $(\varepsilon=1)$ models.

Theorem 3 (Justification of the Serre and Green-Naghdi models). Let $t_{0}>1$, $\varepsilon=\sqrt{\mu}$ (Serre) or $\varepsilon=1$ (Green-Naghdi), and $s \geq t_{0}+2$.

Let also $\underline{T}>0$ and $\left(V_{a p p}^{\mu}, \zeta_{a p p}^{\mu}\right)_{0<\mu<1}$ be bounded in $C\left(\left[0, \frac{\underline{T}}{\varepsilon}\right] ; X^{s+40}\right)$ and assume that $h_{a p p}^{\mu}:=1+\varepsilon\left(\zeta_{a p p}^{\mu}-b\right)$ satisfies (36) at $t=0$. If moreover

$$
\left\{\begin{array}{c}
\left(h_{a p p}^{\mu}+\mu \mathcal{T}\left[h_{a p p}^{\mu}, \varepsilon b\right]\right) \partial_{t} V_{a p p}^{\mu}+h_{a p p}^{\mu} \nabla \zeta_{a p p}^{\mu}+\varepsilon h_{a p p}^{\mu}\left(V_{a p p}^{\mu} \cdot \nabla\right) V_{a p p}^{\mu} \\
+\mu \varepsilon\left[\frac{1}{3} \nabla\left(h_{a p p}^{\mu}{ }^{3} \mathcal{D}_{V_{a p p}^{\mu}}^{\mu} \operatorname{div}\left(V_{a p p}^{\mu}\right)\right)+\mathcal{Q}\left[h_{a p p}^{\mu}, \varepsilon b\right]\left(V_{a p p}^{\mu}\right)\right]=\mu^{2} R_{1}^{\mu} \\
\partial_{t} \zeta_{a p p}^{\mu}+\nabla \cdot\left(h_{a p p}^{\mu} V_{a p p}^{\mu}\right)=\mu^{2} r_{2}^{\mu},
\end{array}\right.
$$

with $\left(R_{1}^{\mu}, r_{2}^{\mu}\right)_{0<\mu<1}$ bounded in $C\left(\left[0, \frac{T}{\varepsilon}\right] ; X^{s+38}\right)$, then there exists $0<T \leq \underline{T}$ and a unique solution $\left(V^{\mu}, \zeta^{\mu}\right)_{0<\mu<1} \in C\left([0, T / \varepsilon]: X^{s+4}\right)$ to (35) with initial conditions $\left(V_{\left.a p p\right|_{t=0}}^{\mu}, \zeta_{\left.a p p\right|_{t=0}}^{\mu}\right)$. Moreover, one has

$$
\sup _{0 \leq t \leq T}\left|\left(V^{\mu}, \zeta^{\mu}\right)-\left(V_{a p p}^{\mu}, \zeta_{a p p}^{\mu}\right)\right| \lesssim \mu^{2} / \varepsilon,
$$

uniformly with respect to $0<\mu<1$. Restricting to small enough values of $\mu$, one can moreover take $T=\underline{T}$.

Remark 10. The existence of a family $\left(V_{a p p}^{\mu}, \zeta_{a p p}^{\mu}\right)_{0<\mu<1}$ having the properties assumed in the theorem is established in [2].

Proof. Since Assumptions 13 have been checked in the proof of Theorem 2 the result is a direct consequence of Corollary 1 .

Acknowledgment: The authors thank B. Texier for fruitful discussions.

\section{REFERENCES}

[1] Serge Alinhac and Patrick Gérard. Opérateurs pseudo-différentiels et théorème de NashMoser. Savoirs Actuels. [Current Scholarship]. InterEditions, Paris, 1991.

[2] B. Alvarez-Samaniego and D. Lannes. Large time existence for $3 d$ water-waves and asymptotics. Technical report, Université Bordeaux I; IMB, 2007.

[3] S. V. Basenkova, N. N. Morozov, and O. P. Pogutse. Dispersive effects in two-dimensional hydrodynamics. Dokl. Akad. Nauk SSSR, 1985.

[4] A. E. Green, N. Laws, and P. M. Naghdi. On the theory of water waves. Proc. Roy. Soc. (London) Ser. A, 338:43-55, 1974.

[5] A. E. Green and P. M. Naghdi. Derivation of equations for wave propagation in water of variable depth. J. Fluid Mech., 1976.

[6] E. Grenier. Pseudo-differential energy estimates of singular perturbations. Comm. Pure Appl. Math., 50(9):821-865, 1997.

[7] Richard S. Hamilton. The inverse function theorem of Nash and Moser. Bull. Amer. Math. Soc. (N.S.), 7(1):65-222, 1982.

[8] G. Iooss, P. I. Plotnikov, and J. F. Toland. Standing waves on an infinitely deep perfect fluid under gravity. Arch. Ration. Mech. Anal., 177(3):367-478, 2005.

[9] J. W. Kim, K. J. Bai, R. C. Ertekin, and W. C. Webster. A strongly-nonlinear model for water waves in water of variable depth: the irrotational green-naghdi model. Journal of Offshore Mechanics and Arctic Engineering, Trans. of ASME, 2003.

[10] David Lannes. Well-posedness of the water-waves equations. J. Amer. Math. Soc., 18(3):605654 (electronic), 2005.

[11] David Lannes. Sharp estimates for pseudo-differential operators with symbols of limited smoothness and commutators. J. Funct. Anal., 232(2):495-539, 2006.

[12] Yi A. Li. A shallow-water approximation to the full water wave problem. Comm. Pure Appl. Math., 59(9):1225-1285, 2006. 
[13] Hans Lindblad. Well posedness for the motion of a compressible liquid with free surface boundary. Comm. Math. Phys., 260(2):319-392, 2005.

[14] Hans Lindblad. Well-posedness for the motion of an incompressible liquid with free surface boundary. Ann. of Math. (2), 162(1):109-194, 2005.

[15] John Nash. The imbedding problem for Riemannian manifolds. Ann. of Math. (2), 63:20-63, 1956.

[16] Markus Poppenberg. Nash-Moser techniques for nonlinear boundary-value problems. Electron. J. Differential Equations, pages No. 54, 33 pp. (electronic), 2003.

[17] Markus Poppenberg, Klaus Schmitt, and Zhi-Qiang Wang. On the existence of soliton solutions to quasilinear Schrödinger equations. Calc. Var. Partial Differential Equations, 14(3):329-344, 2002.

[18] Xavier Saint Raymond. A simple Nash-Moser implicit function theorem. Enseign. Math. (2), 35(3-4):217-226, 1989.

[19] Michael E. Taylor. Partial differential equations. III, volume 117 of Applied Mathematical Sciences. Springer-Verlag, New York, 1997. Nonlinear equations, Corrected reprint of the 1996 original.

[20] Ge Wei, James T. Kirby, Stephan T. Grilli, and Ravishankar Subramanya. A fully nonlinear Boussinesq model for surface waves. I. Highly nonlinear unsteady waves. J. Fluid Mech., 294:71-92, 1995.

Université Bordeaux I; IMB et CNRS UMR 5251, 351 Cours de la Libération, 33405 Talence Cedex, France 